\title{
ROBUST PRICING OF EUROPEAN OPTIONS WITH WAVELETS AND THE CHARACTERISTIC FUNCTION
}

\author{
LUIS ORTIZ-GRACIA AND CORNELIS W. OOSTERLEE
}

\begin{abstract}
We present a novel method for pricing European options based on the wavelet approximation (WA) method and the characteristic function. We focus on the discounted expected payoff pricing formula, and compute it by means of wavelets. We approximate the density function associated to the underlying asset price process by a finite combination of $j$ th order B-splines, and recover the coefficients of the approximation from the characteristic function. Two variants for wavelet approximation will be presented, where the second variant adaptively determines the range of integration. The compact support of a B-splines basis enables us to price options in a robust way, even in cases where Fourier-based pricing methods may show weaknesses. The method appears to be particularly robust for pricing long-maturity options, fat tailed distributions, as well as staircase-like density functions encountered in portfolio loss computations.
\end{abstract}

\section{INTRODUCTION}

The robust and efficient valuation of financial products, like options, by numerical techniques is an interesting recent field in applied mathematics and scientific computing. The best known option pricing partial differential equation (PDE) is without any doubt the Black-Scholes equation, pricing a European (plain vanilla) option under Geometric Brownian Motion asset price dynamics. When considering more realistic asset dynamics, like stochastic volatility or Lévy jump asset models, other option pricing PDEs, or even partial integro-differential equations, will be encountered.

Option pricing is often done by the discounted expected payoff approach, which is nothing but a Green's function integral formulation for the PDEs mentioned above. The connection between the solution of the option pricing PDEs and the discounted expected payoff lies in the Feynman-Kac theorem. In many cases in option pricing, we do not have the Green's function (read, the conditional probability density function for the asset prices) available, but we do have its Fourier transform. This Fourier transform is the characteristic function, and the theory in [Duf00] shows that for affine PDEs the characteristic function can easily be determined. This derivation technique is related to classical theory regarding the use of the Laplace transform for linear PDEs.

Highly efficient numerical pricing techniques in this context are found in the class of Fourier-based numerical integration [Car99, Lor07]. An exponent of this class is the COS method [Fan08], which is based on Fourier cosine expansions, and on the availability of the characteristic function of the asset price dynamics. However, the COS method, although highly efficient, may lack robustness for a number of cases. For call options, for example, it is often recommended to employ the put-call parity relation, and price calls via puts. The integration interval must be defined a priori and the method also shows sensitivity in the choice of the interval for certain underlying processes like CGMY. Furthermore, with the COS method it is non trivial to handle accurately long maturity contracts as roundoff errors are produced in some cases.

Date: January 2013. 
Other techniques that have been employed for pricing options in this context include the Hilbert transform [Fen08], and the Gauss transform [Bro03].

In the present paper we will focus on the application of wavelet techniques in the context of the discounted expected payoff approach. Wavelets have been used before in option pricing [Mat04, Mat05], but then directly as part of the numerical discretization for the option pricing PDEs. In the framework we consider here, the wavelet approximation approach is novel.

We consider the use of Haar wavelets as well as low-order B-spline wavelets. Compared to the use of cosines in the COS method, the computation of wavelet coefficients may be somewhat more involved. However, we will see that the use of wavelets is in particular robust. In terms of the sensitivity with respect to the size of the integration interval, we propose an adaptive wavelet method, avoiding an a-priori choice of the interval. The fact that these wavelets are functions with compact support, enable us to deal properly with roundoff errors that we may encounter when pricing long maturity options. In the context of portfolio loss computations, the use of this wavelet-based method is also of benefit, since we deal with step-like density functions and a Haar basis fits better to the shape of the probability density function than a cosine basis.

Haar wavelets have been used before [Mas11, Ort12a] in the context of portfolio losses, but there the derivations were based on the Laplace transform while we focus on the use of the Fourier transform and the characteristic function in the present paper.

The outline of the paper is as follows: In Section 2 we present the option pricing problem and discuss wavelets and their properties. In Section 3 we explain two different waveletbased approximations for the option pricing problem. The first method is related to a fixed integration range, whereas the second method adaptively determines the suitable range of integration. It is in particular this second method which is free of open parameters and highly robust. Error analysis is subsequently presented in Section 4, and numerical experiments focussing on the method's robustness are found in Section 5. Finally, Section 6 is devoted to conclusions.

\section{Option Pricing With Wavelets}

We consider the risk-neutral valuation formula,

$$
v(x, t)=e^{-r_{\text {int }}(T-t)} \mathbb{E}^{\mathbb{Q}}(v(y, T) \mid x)=e^{-r_{\text {int }}(T-t)} \int_{\mathbb{R}} v(y, T) f(y \mid x) d y,
$$

where $v$ denotes the option value, $T$ is the maturity, $t$ the initial date, $\mathbb{E}^{\mathbb{Q}}$ the expectation operator under the risk-neutral measure $\mathbb{Q}, x$ and $y$ are state variables at time $t$ and $T$, respectively, $f(y \mid x)$ is the probability density of $y$ given $x$ and $r_{\text {int }}$ is the risk-neutral interest rate.

Whereas $f$ is typically not known, the characteristic function of the log-asset price is often known, that is, the Fourier transform of $f$. We represent the payoff as a function of the log-asset price, and denote the log-asset prices by,

$$
x=\log \left(S_{0} / K\right) \quad \text { and } \quad y=\log \left(S_{T} / K\right),
$$

with $S_{t}$ the underlying price at time $t$ and $K$ the strike price. The payoff $v(y, T)$ for European options in log-asset price then reads,

$$
v(y, T)=\left[\alpha \cdot K\left(e^{y}-1\right)\right]^{+}, \quad \text { with }, \quad \alpha= \begin{cases}1, & \text { for a call } \\ -1, & \text { for a put }\end{cases}
$$

We derive the Wavelet Approximation (WA) method for pricing European options by replacing the density function by an approximation with $j^{\text {th }}$ order B-splines at a fixed scale level $m$. 
A natural and convenient way to introduce wavelets is following the notion of multiresolution analysis (MRA). Here we provide the basic definitions and properties regarding MRA and B-spline wavelets, for further information see [Chu92, Chu97].

2.1. Wavelets and Dual Wavelets. We start with the definition of a Riesz basis, as follows,

Definition 1. A countable set $\left\{f_{n}\right\}$ of a Hilbert space is a Riesz basis if every element $f$ of the space can be uniquely written as $f=\sum_{n} c_{n} f_{n}$, and there exist positive constants $A$ and $B$ such that,

$$
A\|f\|^{2} \leq \sum_{n}\left|c_{n}\right|^{2} \leq B\|f\|^{2} .
$$

Definition 2. A function $\psi \in L^{2}(\mathbb{R})$ is called an $\mathcal{R}$-function if $\left\{\psi_{j, k}\right\}$ defined as $\psi_{j, k}(x):=$ $2^{j / 2} \psi\left(2^{j} x-k\right), j, k \in \mathbb{Z}$, is a Riesz basis of $L^{2}(\mathbb{R})$.

If we assume that $\psi$ is an $\mathcal{R}$-function, then there exists a unique Riesz basis $\left\{\psi^{l, m}\right\}$ of $L^{2}(\mathbb{R})$, which is dual to $\left\{\psi_{j, k}\right\}$ in the sense that $\left\langle\psi_{j, k}, \psi^{l, m}\right\rangle=\delta_{j, l} \delta_{k, m}$, for all $j, k, l, m \in \mathbb{Z}$, and $\delta_{p, q}$ is the Kronecker delta defined in the usual way as,

$$
\delta_{p, q}= \begin{cases}1, & p=q, \\ 0, & \text { otherwise. }\end{cases}
$$

With the above definitions, we can give the definition of wavelets.

Definition 3. An $\mathcal{R}$-function $\psi \in L^{2}(\mathbb{R})$ is called an $\mathcal{R}$-wavelet, or simply a wavelet, if there exists a function $\widetilde{\psi} \in L^{2}(\mathbb{R})$, such that $\left\{\psi_{j, k}\right\}$ and $\left\{\widetilde{\psi}_{j, k}\right\}$ defined as $\left\{\widetilde{\psi}_{j, k}\right\}=2^{j / 2} \widetilde{\psi}\left(2^{j} x-\right.$ $k), j, k \in \mathbb{Z}$, are dual bases of $L^{2}(\mathbb{R})$. If $\psi$ is a wavelet, then $\widetilde{\psi}$ is called a dual wavelet corresponding to $\psi$.

Some definitions relevant to our present work are given below,

Definition 4. Let $\psi$ be the wavelet function in Definition 3.

(i) A wavelet $\psi$ is said to have a vanishing moment of order $s$ if $\int_{\mathbb{R}} x^{p} \psi(x) d x=0, p=$ $0, \ldots, s-1$. All wavelets must satisfy this condition for $p=0$.

(ii) A wavelet $\psi$ is an orthogonal $(O N)$ wavelet if the family $\left\{\psi_{j, k}\right\}$ forms an orthonormal basis of $L^{2}(\mathbb{R})$, that is, $\left\langle\psi_{s, t}, \psi_{u, v}\right\rangle=\delta_{s, u} \delta_{t, v}$, for all $s, u, t, v \in \mathbb{Z}$.

(iii) A wavelet $\psi$ is called a semi-orthogonal (SO) wavelet if the family $\left\{\psi_{j, k}\right\}$ satisfies, $\left\langle\psi_{s, t}, \psi_{u, v}\right\rangle=0, s \neq u$, for all $s, u, t, v \in \mathbb{Z}$.

A dual wavelet $\widetilde{\psi}$ is unique and is itself a wavelet. The pair $(\psi, \widetilde{\psi})$ is symmetric in the sense that $\psi$ is also the dual wavelet of $\widetilde{\psi}$. If $\psi$ is an orthogonal wavelet, then it is self-dual in the sense of $\widetilde{\psi} \equiv \psi$. Moreover, it is important to emphasize that if $f \in L^{2}(\mathbb{R})$ then,

$$
f(x)=\sum_{j, k=-\infty}^{+\infty}\left\langle f, \psi_{j, k}\right\rangle \widetilde{\psi}_{j, k}=\sum_{j, k=-\infty}^{+\infty}\left\langle f, \widetilde{\psi}_{j, k}\right\rangle \psi_{j, k} .
$$

For each $j \in \mathbb{Z}$, let $W_{j}$ denote the closure of the linear span of $\left\{\psi_{j, k}: k \in \mathbb{Z}\right\}$, namely, $W_{j}:=\operatorname{clos}_{L^{2}(\mathbb{R})}\left\langle\psi_{j, k}: k \in \mathbb{Z}\right\rangle$. Then, $L^{2}(\mathbb{R})$ can be decomposed as a direct sum of the spaces $W_{j}$,

$$
L^{2}(\mathbb{R})=\sum_{j \in \mathbb{Z}}^{\bullet} W_{j}:=\cdots+W_{-1}+W_{0}+W_{1} \dot{+} \cdots,
$$

in the sense that every function $f \in L^{2}(\mathbb{R})$ has a unique decomposition,

$$
f(x)=\cdots+g_{-1}(x)+g_{0}(x)+g_{1}(x)+\cdots,
$$


where $g_{j} \in W_{j}, j \in \mathbb{Z}$.

If $\psi$ is an orthogonal wavelet, then the subspaces $W_{j}$ of $L^{2}(\mathbb{R})$ are mutually orthogonal and consequently, the direct sum in (2) becomes an orthogonal sum,

$$
L^{2}(\mathbb{R})=\bigoplus_{j \in \mathbb{Z}} W_{j}:=\cdots \oplus W_{-1} \oplus W_{0} \oplus W_{1} \oplus \cdots .
$$

Obviously, every SO wavelet generates an orthogonal decomposition (3) of $L^{2}(\mathbb{R})$, and every $\mathrm{ON}$ wavelet is also an $\mathrm{SO}$ wavelet.

2.2. Multiresolution Analysis, Scaling Functions and B-Splines. Any wavelet, semiorthogonal or not, generates a direct sum decomposition $(2)$ of $L^{2}(\mathbb{R})$. For each $j \in \mathbb{Z}$, let us consider the closed subspaces,

$$
V_{j}=\cdots \dot{+} W_{j-2} \dot{+} W_{j-1}, \quad j \in \mathbb{Z}
$$

of $L^{2}(\mathbb{R})$. These subspaces have the following properties,

(i) $\cdots \subset V_{-1} \subset V_{0} \subset V_{1} \subset \cdots$,

(ii) $\operatorname{clos}_{L^{2}}\left(\bigcup_{j \in \mathbb{Z}} V_{j}\right)=L^{2}(\mathbb{R})$,

(iii) $\bigcap_{j \in \mathbb{Z}} V_{j}=\{0\}$,

(iv) $V_{j+1}=V_{j} \dot{+} W_{j}, j \in \mathbb{Z}$,

(v) $f(x) \in V_{j} \Leftrightarrow f(2 x) \in V_{j+1}, j \in \mathbb{Z}$.

Observe that every function $f \in L^{2}(\mathbb{R})$ can be approximated as closely as desirable by its projections $P_{j} f$ in $V_{j}$ as described by (ii).

If the reference subspace $V_{0}$, say, is generated by a single function $\phi \in L^{2}(\mathbb{R})$ in the sense that,

$$
V_{0}:=\operatorname{clos}_{L^{2}(\mathbb{R})}\left\langle\phi_{0, k}: k \in \mathbb{Z}\right\rangle,
$$

where $\phi_{j, k}:=2^{j / 2} \phi\left(2^{j} x-k\right)$, then all subspaces $V_{j}$ are also generated by the same $\phi$, namely,

$$
V_{j}:=\operatorname{clos}_{L^{2}(\mathbb{R})}\left\langle\phi_{j, k}: k \in \mathbb{Z}\right\rangle, \quad j \in \mathbb{Z} .
$$

Definition 5. A function $\phi \in L^{2}(\mathbb{R})$ is said to generate a multiresolution analysis (MRA) if it generates a nested sequence of closed subspaces $V_{j}$ that satisfy (i), (ii), (iii) and $(v)$ in the sense of (4), such that $\left\{\phi_{0, k}\right\}$ forms a Riesz basis of $V_{0}$. If $\phi$ generates a MRA, then $\phi$ is called a scaling function.

Typical examples of scaling functions $\phi$ are the $j^{\text {th }}$ order cardinal $B$-splines, $N_{j}(x)$, defined recursively by a convolution,

$$
N_{j}(x)=\int_{-\infty}^{\infty} N_{j-1}(x-t) N_{0}(t) d t=\int_{0}^{1} N_{j-1}(x-t) d t, \quad j \geq 1,
$$

where,

$$
N_{0}(x)=\chi_{[0,1)}(x)= \begin{cases}1, & \text { if } x \in[0,1) \\ 0, & \text { otherwise }\end{cases}
$$

Alternatively,

$$
N_{j}(x)=\frac{x}{j} N_{j-1}(x)+\frac{j+1-x}{j} N_{j-1}(x-1), \quad j \geq 1 .
$$

We note that cardinal $B$-spline functions are compactly supported, since the support of the $j^{\text {th }}$ order $B$-spline function $N_{j}$ is $[0, j+1]$, and they have as the Fourier transform,

$$
\widehat{N_{j}}(w)=\left(\frac{1-e^{-i w}}{i w}\right)^{j+1} .
$$


To describe the space $V_{0}$ that $N_{j}$ generates, we define $\pi_{n}$ as the collection of all polynomials of degree at most $n$, and $C^{n}$ denotes the collection of all functions $f$ such that $f, f^{(1)}, \ldots, f^{(n)}$ are continuous everywhere.

The subspace $V_{0}$ generated by $N_{j}$ consists of all functions $f \in C^{j-1} \cap L^{2}(\mathbb{R})$ such that the restriction of each function $f$ to any interval $[k, k+1), k \in \mathbb{Z}$, is in $\pi_{j}$. From property (v) of an MRA, we can now identify all other subspaces $V_{j}$, namely,

$$
V_{j}=\left\{f \in C^{s-1} \cap L^{2}(\mathbb{R}):\left.f\right|_{\left[\frac{k}{2 j}, \frac{k+1}{2^{j}}\right)} \in \pi_{s}, k \in \mathbb{Z}\right\} .
$$

Since splines are only piecewise polynomial functions, they are very easy to implement in a computer code.

From the nested sequence of splines subspaces $V_{j}$, we have the orthogonal complementary subspaces $W_{j}$, such that, $V_{j+1}=V_{j} \oplus W_{j}, j \in \mathbb{Z}$. Just as the B-spline of order $s$ is the minimally supported generator of $\left\{V_{j}\right\}$ we can find the minimally supported $\psi^{s} \in W_{0}$ that generates the mutually orthogonal subspaces $W_{j}$. These compactly supported functions will be called B-wavelets of order $s$. In Chapter 6 in [Chu92], explicit formulas for all $\psi^{s}$ and their duals are derived.

In this paper we consider $\phi^{j}=N_{j}$ as the scaling function which generates a MRA (see Figure 1) and we restrict ourselves to the orders $j=0,1$. Clearly, for $j=0$ we have the scaling function of the Haar wavelet system. We also remark that from the previous discussions, for every function $f_{m} \in V_{m}$, there exists a unique sequence $\left\{c_{m, k}^{j}\right\}_{k \in \mathbb{Z}} \in l^{2}(\mathbb{Z})$, such that,

$$
f_{m, j}(x)=\sum_{k \in \mathbb{Z}} c_{m, k}^{j} \phi_{m, k}^{j}(x)
$$

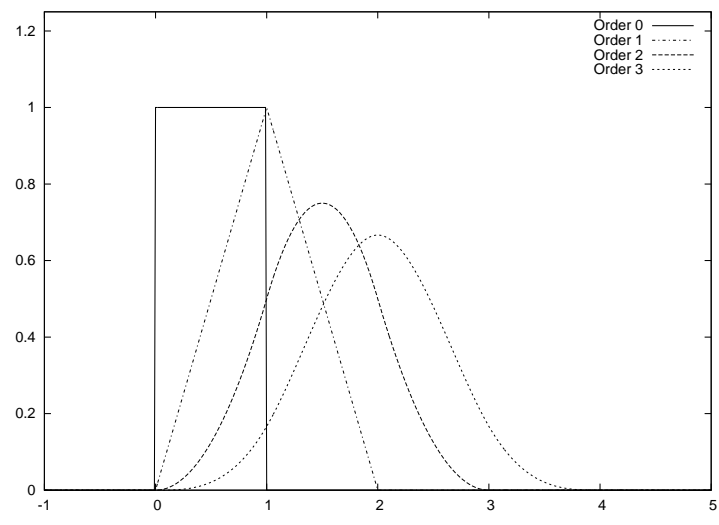

Figure 1: Cardinal B-splines of orders $j=0,1,2,3$.

In this paper we present two different methods for approximating the density function in (1). In the first method we restrict ourselves to a bounded interval $[a, b]$,

$$
f_{m, j}^{c}(x)=\sum_{k=0}^{(j+1) \cdot\left(2^{m}-1\right)} c_{m, k}^{j} \phi_{m, k}^{j}\left((j+1) \cdot \frac{x-a}{b-a}\right), \quad j \geq 0,
$$

while in the second method we carry out the approximation on the entire real line as in (5). For this reason, it is convenient to give a short review about B-splines on a bounded interval. We refer the reader to [Chu97] for a detailed description of scaling functions on a bounded interval. 
Let us assume that $[0, n], n \in \mathbb{N}$, is the working interval. We must distinguish between interior B-splines and boundary B-splines. We have,

$$
N_{j}(x-k), \quad k=0, \ldots, n-j-1 .
$$

These are the interior B-splines for the bounded interval $[0, n]$. The remaining B-splines,

$$
\begin{cases}N_{j}(x-k), & k=-j, \ldots,-1, \text { and } \\ N_{j}(x-k), & k=n-j, \cdots, n-1,\end{cases}
$$

are the boundary B-splines for the interval $[0, n]$. Here, the first group is for the boundary $x=0$, while the second group is for the boundary $x=n$.

\section{The Wavelet Approximation Method}

We present two different methods to recover a function $f$ from its Fourier transform. The first method relies on fixing a priori the interval for the approximation and then using Bsplines wavelets on this bounded interval, while the second method performs the approximation in an adaptive way, computing the domain of the approximation such that a predefined tolerance error is met. Later on, we will apply both methods to the option pricing problem and we will discuss the pros and cons of each of them.

3.1. Approximation in a fixed interval $[a, b]$ : the $\mathbf{W A}^{[a, b]} \operatorname{method}$. Let us consider a function $f \in L^{2}(\mathbb{R})$ and its Fourier transform, whenever it exists:

$$
\widehat{f}(w)=\int_{-\infty}^{+\infty} e^{-i w x} f(x) d x .
$$

Since $f \in L^{2}(\mathbb{R})$ we can expect that $f$ decays to zero, so it can be well approximated in a finite interval $[a, b]$ by,

$$
f^{c}(x)= \begin{cases}f(x), & \text { if } x \in[a, b] \\ 0, & \text { otherwise }\end{cases}
$$

Following the theory of MRA in a bounded interval, we can approximate $f^{c}(x) \simeq f_{m, j}^{c}(x)$ for all $x \in[a, b]$, where,

$$
f_{m, j}^{c}(x)=\sum_{k=0}^{(j+1) \cdot\left(2^{m}-1\right)} c_{m, k}^{j} \phi_{m, k}^{j}\left((j+1) \cdot \frac{x-a}{b-a}\right), \quad j \geq 0,
$$

with convergence in $L^{2}$-norm. Note that we are not considering the left and right boundary scaling functions. For sake of simplicity, we set the coefficients corresponding to the boundary basis functions equal to zero. Observe that in the case that $j=0$ (Haar wavelets) there are no boundary functions.

The main idea behind the Wavelet Approximation method is to approximate $\widehat{f}$ by $\widehat{f}_{m, j}^{c}$ and then to compute the coefficients $c_{m, k}^{j}$ by inverting the Fourier Transform. Proceeding this way, we have,

$$
\begin{aligned}
\widehat{f}(w) & =\int_{-\infty}^{+\infty} e^{-i w x} f(x) d x \simeq \int_{-\infty}^{+\infty} e^{-i w x} f_{m, j}^{c}(x) d x \\
& =\sum_{k=0}^{(j+1) \cdot\left(2^{m}-1\right)} c_{m, k}^{j}\left(\int_{-\infty}^{+\infty} e^{-i w x} \phi_{m, k}^{j}\left((j+1) \cdot \frac{x-a}{b-a}\right) d x\right) .
\end{aligned}
$$


Introducing a change of variables, $y=(j+1) \cdot \frac{x-a}{b-a}$, gives us,

$$
\begin{aligned}
\widehat{f}(w) & \simeq \frac{b-a}{j+1} \cdot e^{-i a w} \sum_{k=0}^{(j+1) \cdot\left(2^{m}-1\right)} c_{m, k}^{j} \int_{-\infty}^{+\infty} e^{-i w \frac{b-a}{j+1} y} \phi_{m, k}^{j}(y) d y \\
& =\frac{b-a}{j+1} \cdot e^{-i a w} \sum_{k=0}^{(j+1) \cdot\left(2^{m}-1\right)} c_{m, k}^{j} \widehat{\phi}_{m, k}^{j}\left(\frac{b-a}{j+1} \cdot w\right) .
\end{aligned}
$$

Finally, taking into account that $\widehat{\phi}_{m, k}^{j}(\xi)=2^{-\frac{m}{2}} \widehat{\phi}^{j}\left(\frac{\xi}{2^{m}}\right) e^{-i \frac{k}{2^{m}} \xi}$ and performing a change of variables, $z=e^{-i \frac{b-a}{2^{m}(j+1)} w}$, we find,

$$
\widehat{f}\left(\frac{2^{m}(j+1)}{b-a} i \cdot \log (z)\right) \simeq 2^{-\frac{m}{2}} \frac{b-a}{j+1} \cdot z^{\frac{2^{m}(j+1) a}{b-a}} \widehat{\phi^{j}}(i \cdot \log (z)) \sum_{k=0}^{(j+1) \cdot\left(2^{m}-1\right)} c_{m, k}^{j} z^{k} .
$$

If we define,

$$
P_{m, j}(z):=\sum_{k=0}^{(j+1) \cdot\left(2^{m}-1\right)} c_{m, k}^{j} z^{k} \text { and } Q_{m, j}(z):=\frac{2^{\frac{m}{2}}(j+1) z^{-\frac{2^{m}(j+1) a}{b-a}} \widehat{f}\left(\frac{2^{m}(j+1)}{b-a} i \cdot \log (z)\right)}{(b-a) \widehat{\phi^{j}}(i \cdot \log (z))},
$$

then, according to the previous formula (7), we have,

$$
P_{m, j}(z) \simeq Q_{m, j}(z) \text {. }
$$

Since $P_{m, j}(z)$ is a polynomial, it is (in particular) analytic inside a disc of the complex plane $\{z \in \mathbb{C}:|z|<r\}$ for $r>0$. We can obtain expressions for the coefficients $c_{m, k}^{j}$ by means of Cauchy's integral formula. This is,

$$
c_{m, k}^{j}=\frac{1}{2 \pi i} \int_{\gamma} \frac{P_{m, j}(z)}{z^{k+1}} d z, \quad k=0, \ldots,(j+1) \cdot\left(2^{m}-1\right),
$$

where $\gamma$ denotes a circle of radius $r, r>0$, about the origin.

Considering now the change of variables $z=r e^{i u}, r>0$, gives us,

$$
c_{m, k}^{j}=\frac{1}{2 \pi r^{k}} \int_{0}^{2 \pi} \frac{P_{m, j}\left(r e^{i u}\right)}{e^{i k u}} d u
$$

where $k=0, \ldots,(j+1) \cdot\left(2^{m}-1\right)$.

Then, we can further expand expression (9) by (see Appendix A for a detailed proof),

$$
c_{m, 0}^{j}=\frac{1}{\pi} \int_{0}^{\pi} \Re\left(P_{m, j}\left(r e^{i u}\right)\right) d u,
$$

and,

$$
c_{m, k}^{j}=\frac{2}{\pi r^{k}} \int_{0}^{\pi} \Re\left(P_{m, j}\left(r e^{i u}\right)\right) \cos (k u) d u, \quad k=1, \ldots,(j+1) \cdot\left(2^{m}-1\right) .
$$

On the other side, since $\widehat{\phi}^{j}(i \cdot \log (z))=\left(\frac{z-1}{\log (z)}\right)^{j+1}$, we have,

$$
Q_{m, j}(z)=\frac{2^{\frac{m}{2}}(j+1) z^{-\frac{2^{m}(j+1) a}{b-a}} \widehat{f}\left(\frac{2^{m}(j+1)}{b-a} i \cdot \log (z)\right)(\log (z))^{j+1}}{(b-a)(z-1)^{j+1}},
$$

and it has a pole at $z=1$. Finally, making use of (8) and taking into account the former observation, we can exchange $P_{m, j}$ by $Q_{m, j}$ in (10) and (11) to obtain, respectively,

$$
c_{m, 0}^{j} \simeq \frac{1}{\pi} \int_{0}^{\pi} \Re\left(Q_{m, j}\left(r e^{i u}\right)\right) d u,
$$


and,

$$
c_{m, k}^{j} \simeq \frac{2}{\pi r^{k}} \int_{0}^{\pi} \Re\left(Q_{m, j}\left(r e^{i u}\right)\right) \cos (k u) d u, \quad k=1, \ldots,(j+1) \cdot\left(2^{m}-1\right),
$$

where $r \neq 1$ is a positive real number.

In practice, both integrals in (12) and (13) are computed by means of the Trapezoidal Rule, and we can define,

$$
I(k)=\int_{0}^{\pi} \Re\left(Q_{m, j}\left(r e^{i u}\right)\right) \cos (k u) d u
$$

and,

$$
I(k ; h)=\frac{h}{2}\left(Q_{m, j}(r)+(-1)^{k} Q_{m, j}(-r)+2 \sum_{j=1}^{M-1} \Re\left(Q_{m, j}\left(r e^{i h_{s}}\right)\right) \cos \left(k h_{s}\right)\right),
$$

where $h=\frac{\pi}{M}$ and $h_{s}=s h$ for all $s=0, \ldots, M$. Proceeding this way we find,

$$
\begin{aligned}
c_{m, k}^{j} & \simeq \frac{2}{\pi r^{k}} I(k) \simeq \frac{2}{\pi r^{k}} I(k ; h) \\
& =\frac{1}{M r^{k}}\left(Q_{m, j}(r)+(-1)^{k} Q_{m, j}(-r)+2 \sum_{s=1}^{M-1} \Re\left(Q_{m, j}\left(r e^{i h_{s}}\right)\right) \cos \left(k h_{s}\right)\right),
\end{aligned}
$$

where $k=1, \ldots,(j+1) \cdot\left(2^{m}-1\right)$.

3.2. Approximation in $\mathbb{R}$ : the $\mathbf{W A}^{\mathbb{R}}$ method. Let $f$ be a function in $L^{2}(\mathbb{R})$.

Following the theory of MRA in $L^{2}(\mathbb{R})$, we can approximate $f(x)$ by $f_{m, j}(x)$ in $(5)$ for all $x \in \mathbb{R}$, where,

$$
f_{m, j}(x)=\sum_{k \in \mathbb{Z}} c_{m, k}^{j} \phi_{m, k}^{j}(x), \quad j \geq 0,
$$

with convergence in the $L^{2}$-norm. Note that the coefficients $c_{m, k}^{j}$ are different from those in Section 3.1. For sake of clarity, we however keep the same notation as in the previous section.

Taking into account that $f$ decays to zero, we truncate $f_{m, j}$ to obtain,

$$
f_{m, j}^{t r}(z)=\sum_{k=k_{1}}^{k_{2}} c_{m, k}^{j} \phi_{m, k}^{j}(x)
$$

where $k_{1}<k_{2}, k_{1}, k_{2} \in \mathbb{Z}$ are chosen such that,

$$
f_{m, j}\left(\frac{k}{2^{m}}\right) \leq f_{m, j}\left(\frac{k_{1}}{2^{m}}\right)<\epsilon_{\mathrm{tol}}, \quad \text { for all } \quad k \leq k_{1},
$$

and,

$$
f_{m, j}\left(\frac{k}{2^{m}}\right) \leq f_{m, j}\left(\frac{k_{2}}{2^{m}}\right)<\epsilon_{\mathrm{tol}}, \quad \text { for all } \quad k \geq k_{2},
$$

where $\epsilon_{\mathrm{tol}}$ is a predefined tolerance error such that $f_{m, j}(x)<\epsilon_{\mathrm{tol}}$ for all $x \in \mathbb{R} \backslash\left[k_{1}, k_{2}\right]$.

Substituting (17) into the Fourier transform expression (6), making the change of variables $z=e^{-i \frac{w}{2^{m}}}$ and following the steps as in the previous section, gives us,

$$
\widehat{f}\left(2^{m} i \cdot \log (z)\right) \simeq 2^{-\frac{m}{2}} \cdot \widehat{\phi^{j}}(i \cdot \log (z)) \sum_{k=k_{1}}^{k_{2}} c_{m, k}^{j} z^{k}
$$


If we define,

$$
P_{m, j}^{*}(z):=\sum_{k=k_{1}}^{k_{2}} c_{m, k}^{j} z^{k} \quad \text { and } \quad Q_{m, j}^{*}(z):=\frac{2^{\frac{m}{2}} \widehat{f}\left(2^{m} i \cdot \log (z)\right)}{\widehat{\phi}^{j}(i \cdot \log (z))},
$$

then, according to the previous formula, we have,

$$
P_{m, j}^{*}(z) \simeq Q_{m, j}^{*}(z) \text {. }
$$

Now, if we consider the polynomial of degree $k_{2}-k_{1}$,

$$
P_{m, j}(z):=z^{-k_{1}} \cdot P_{m, j}^{*}(z)=\sum_{k=0}^{k_{2}-k_{1}} c_{m, k_{1}+k} z^{k},
$$

we can approximate it by $Q_{m, j}(z)$ as,

$$
P_{m, j}(z) \simeq Q_{m, j}(z)
$$

where, $Q_{m, j}(z)=z^{-k_{1}} \cdot Q_{m, j}^{*}(z)$.

Finally, we can derive the coefficients of the approximation following the same steps as in the $\mathrm{WA}^{[a, b]}$ method to obtain,

$$
c_{m, k_{1}}^{j} \simeq \frac{1}{\pi} \int_{0}^{\pi} \Re\left(Q_{m, j}\left(r e^{i u}\right)\right) d u,
$$

and,

$$
c_{m, k_{1}+k}^{j} \simeq \frac{2}{\pi r^{k}} \int_{0}^{\pi} \Re\left(Q_{m, j}\left(r e^{i u}\right)\right) \cos (k u) d u, \quad k=1, \ldots, k_{2}-k_{1}
$$

3.3. Coefficients $V_{m, k}^{j, \alpha}$ for Plain Vanilla Options. Now, we return to the option pricing problem and we consider option valuation formula (1). From now on, we assume that the density functions are $L^{2}(\mathbb{R})$ functions. Since we can expect that the density function $f(y \mid x)$ rapidly decays to zero, we can apply the methods explained in Section 3.1 and Section 3.2.

We must distinguish between the two methods presented formerly,

- WA ${ }^{[a, b]}$ method. We first truncate the infinity integration range to $[a, b] \subset \mathbb{R}$ without loosing significant accuracy and then approximate the density function,

$$
\begin{gathered}
v^{c}(x, t)=e^{-r_{\mathrm{int}}(T-t)} \int_{a}^{b} v(y, T) f(y \mid x) d y, \\
v_{m, j}^{c}(x, t)=e^{-r_{\mathrm{int}}(T-t)} \int_{a}^{b} v(y, T) f_{m, j}^{c}(y \mid x) d y,
\end{gathered}
$$

where,

$$
f_{m, j}^{c}(y \mid x)=\sum_{k=0}^{(j+1) \cdot\left(2^{m}-1\right)} c_{m, k}^{j}(x) \phi_{m, k}^{j}\left((j+1) \cdot \frac{y-a}{b-a}\right) .
$$

We then expand the expression (19) to obtain,

$$
v(x, t) \simeq v_{m, j}^{c}(x, t)=e^{-r_{\text {int }}(T-t)} \sum_{k=0}^{(j+1) \cdot\left(2^{m}-1\right)} c_{m, k}^{j}(x) \cdot V_{m, k}^{j, \alpha},
$$

where,

$$
V_{m, k}^{j, \alpha}:=\int_{a}^{b} v(y, T) \phi_{m, k}^{j}\left((j+1) \cdot \frac{y-a}{b-a}\right) d y
$$


- $\mathbf{W A}^{\mathbb{R}}$ method. Here, we first approximate the density function by a series of scaling functions in $L^{2}(\mathbb{R})$ and then truncate the series expansion, i.e.,

$$
\begin{aligned}
& v_{m, j}(x, t)=e^{-r_{\mathrm{int}}(T-t)} \int_{\mathbb{R}} v(y, T) f_{m, j}(y \mid x) d y, \\
& v_{m, j}^{t r}(x, t)=e^{-r_{\mathrm{int}}(T-t)} \int_{\mathbb{R}} v(y, T) f_{m, j}^{t r}(y \mid x) d y,
\end{aligned}
$$

where,

$$
f_{m, j}^{t r}(y \mid x):=\sum_{k=k_{1}}^{k_{2}} c_{m, k}^{j}(x) \phi_{m, k}^{j}(y)
$$

Then,

$$
v(x, t) \simeq v_{m, j}^{t r}(x, t)=e^{-r_{\mathrm{int}}(T-t)} \sum_{k=k_{1}}^{k_{2}} c_{m, k}^{j}(x) \cdot V_{m, k}^{j, \alpha}
$$

where,

$$
V_{m, k}^{j, \alpha}:=\int_{\mathbb{R}} v(y, T) \phi_{m, k}^{j}(y) d y
$$

Remark 1. Once we have an expression for the scaling coefficients $c_{m, k}^{j}(x)$ and the payoff coeffcients $V_{m, k}^{j, \alpha}$, the Greeks can be easily derived by differentiation.

3.3.1. Coefficients with Haar Wavelets. We first consider $j=0$. Then, the following Proposition holds,

Proposition 1. Let us define $\Delta_{m}=\frac{b-a}{2^{m}}, \beta_{k}=a+k \Delta_{m}, \gamma_{k}=\beta_{k}+\Delta_{m}, \delta_{k}=\max \left(0, \beta_{k}\right)$ and $\zeta_{k}=\min \left(0, \gamma_{k}\right)$. Assuming that $a<0<b$, then the coefficients computed by the $W A^{[a, b]}$ method are as follows,

$$
\begin{aligned}
V_{m, k}^{0,1} & = \begin{cases}2^{m / 2} K\left(e^{\gamma_{k}}-e^{\delta_{k}}+\delta_{k}-\gamma_{k}\right), & \gamma_{k}>0, \\
0, & \text { otherwise. }\end{cases} \\
V_{m, k}^{0,-1} & = \begin{cases}2^{m / 2} K\left(e^{\beta_{k}}-e^{\zeta_{k}}+\zeta_{k}-\beta_{k}\right), & \beta_{k}<0, \\
0, & \text { otherwise. }\end{cases}
\end{aligned}
$$

for $k=0, \ldots, 2^{m}-1$.

If $a<b<0$ then, $V_{m, k}^{0,1}=0, V_{m, k}^{0,-1}=2^{m / 2} K\left(e^{\beta_{k}}-e^{\gamma_{k}}+\gamma_{k}-\beta_{k}\right)$, for $k=0, \ldots, 2^{m}-1$. And conversely, if $0<a<b$ then, $V_{m, k}^{0,-1}=0, V_{m, k}^{0,1}=2^{m / 2} K\left(e^{\gamma_{k}}-e^{\beta_{k}}+\beta_{k}-\gamma_{k}\right)$, for $k=0, \ldots, 2^{m}-1$.

Proof. We consider three cases separately,

- Case $a<0<b$. We first consider call options, i.e., the case that $\alpha=1$. By definition,

$$
V_{m, k}^{0,1}:=\int_{a}^{b}\left[K\left(e^{y}-1\right)\right]^{+} \phi_{m, k}^{0}\left(\frac{y-a}{b-a}\right) d y=\int_{0}^{b} K\left(e^{y}-1\right) \phi_{m, k}^{0}\left(\frac{y-a}{b-a}\right) d y .
$$

Taking into account that,

$$
\phi_{m, k}^{0}\left(\frac{y-a}{b-a}\right)= \begin{cases}2^{m / 2}, & \frac{k}{2^{m}} \leq \frac{y-a}{b-a}<\frac{k+1}{2^{m}} \\ 0, & \text { otherwise }\end{cases}
$$

we have,

$$
V_{m, k}^{0,1}=\int_{[0, b] \cap\left[\beta_{k}, \gamma_{k}\right]} 2^{m / 2} K\left(e^{y}-1\right) d y=\left\{\begin{array}{ll}
\int_{\delta_{k}}^{\gamma_{k}} 2^{m / 2} K\left(e^{y}-1\right) d y, & \gamma_{k}>0, \\
0, & \text { otherwise. }
\end{array} .\right.
$$


For put options, i.e., $\alpha=-1$, we find,

$$
\begin{aligned}
V_{m, k}^{0,-1} & =\int_{a}^{0}-K\left(e^{y}-1\right) \phi_{m, k}^{0}\left(\frac{y-a}{b-a}\right) d y=\int_{[a, 0] \cap\left[\beta_{k}, \gamma_{k}\right]}-2^{m / 2} K\left(e^{y}-1\right) d y \\
& = \begin{cases}\int_{\beta_{k}}^{\zeta_{k}}-2^{m / 2} K\left(e^{y}-1\right) d y, & \beta_{k}<0, \\
0, & \text { otherwise. }\end{cases}
\end{aligned}
$$

- Case $a<b<0$. In this case, supp $\phi_{m, k}^{0} \subset(-\infty, 0)$ and for call options $v(y, T)=0, y \leq$ 0 . Then, $V_{m, k}^{0,1}=0, k=0, \ldots, 2^{m}-1$. For put options $v(y, T)>0, y \leq 0$, and therefore $V_{m, k}^{0,-1}=\int_{[a, b] \cap\left[\beta_{k}, \gamma_{k}\right]}-2^{m / 2} K\left(e^{y}-1\right) d y=\int_{\beta_{k}}^{\gamma_{k}}-2^{m / 2} K\left(e^{y}-1\right) d y, k=0, \ldots, 2^{m}-1$.

- Case $0<a<b$. In this case, supp $\phi_{m, k}^{0} \subset(0,+\infty)$ and for call options $v(y, T)>$ $0, y>0$. Then, $V_{m, k}^{0,1}=\int_{[a, b] \cap\left[\beta_{k}, \gamma_{k}\right]} 2^{m / 2} K\left(e^{y}-1\right) d y=\int_{\beta_{k}}^{\gamma_{k}} 2^{m / 2} K\left(e^{y}-1\right) d y, k=$ $0, \ldots, 2^{m}-1$. For put options $v(y, T)=0, y \geq 0$, and therefore $V_{m, k}^{0,-1}=0, k=$ $0, \ldots, 2^{m}-1$.

Corollary 1. The prices for a put and for a call can be obtained simultaneously with a WA ${ }^{[a, b]}$ method at resolution level $m$, by computing $2^{m}$ coefficients $c_{m, k}^{0}$ and $2^{m}$ coefficients $V_{m, k}^{0, \alpha}$.

Proof. For the cases $a<b<0$ and $0<a<b$ the statement holds immediately. We consider the non-trivial case $a<0<b$. By Proposition 1 we just need to compute the coefficients such that $\gamma_{k}>0$ and $\beta_{k}<0$. Following the same notation as before,

$$
\gamma_{k}>0 \Leftrightarrow \beta_{k}+\Delta_{m}>0 \Leftrightarrow a+k \Delta_{m}+\Delta_{m}>0 \Leftrightarrow k+1>-\frac{a}{\Delta_{m}},
$$

and,

$$
\beta_{k}<0 \Leftrightarrow a+k \Delta_{m}<0 \Leftrightarrow k<-\frac{a}{\Delta_{m}}
$$

Since $-\frac{a}{\Delta_{m}}>0$ then,

$$
\gamma_{k}>0 \Leftrightarrow\left\lfloor-\frac{a}{\Delta_{m}}\right\rfloor \leq k \leq 2^{m}-1, \quad \text { and, } \quad \beta_{k}<0 \Leftrightarrow 0 \leq k \leq\left\lfloor-\frac{a}{\Delta_{m}}\right\rfloor,
$$

where $\lfloor x\rfloor$ denotes the greatest integer less than or equal to $x$, and this completes the proof.

Remark 2. Digital options are popular in the financial markets for hedging and speculation. They are also important to financial engineers as building blocks for constructing more complex option products. Here, we consider the payoff of a cash-or-nothing call option as an example, which has value 0 if $S_{T} \leq K$ and $K$ if $S_{T}>K$. For this contract, the $V_{m, k}^{0,1}$ coefficients, like (22), can also be obtained analytically with the $W A^{[a, b]}$ method as follows (we assume that $a<0<b)$,

$$
V_{m, k}^{0,1}= \begin{cases}2^{m / 2} K\left(\delta_{k}-\gamma_{k}\right), & \gamma_{k}>0 \\ 0, & \text { otherwise }\end{cases}
$$

Remark 3. If we define $a:=\frac{k_{1}}{2^{m}}$ and $\Delta_{m}:=\frac{1}{2^{m}}$ then the formulae (21) and (23) also hold to compute the coefficients $V_{m, k}^{0, \alpha}$ by the $W A^{\mathbb{R}}$ method. Moreover, we can price a put and a call simultaneously by computing $k_{2}-k_{1}+1$ coefficients $c_{m, k}^{0}$ and $k_{2}-k_{1}+1$ coefficients $V_{m, k}^{0, \alpha}$. 
3.3.2. Coefficients with Linear B-Splines. We consider $j=1$. Then, the following proposition holds,

Proposition 2. Let us define $\bar{\Delta}_{m}=\frac{b-a}{2^{m+1}}, \bar{\beta}_{k}=a+k \bar{\Delta}_{m}, \bar{\gamma}_{k}=\bar{\beta}_{k}+2 \bar{\Delta}_{m}, \kappa=\frac{\bar{\beta}_{k}+\bar{\gamma}_{k}}{2}, \iota_{k}=$ $\max (0, \kappa), \lambda_{k}=\min (0, \kappa), \bar{\delta}_{k}=\max \left(0, \bar{\beta}_{k}\right), \bar{\zeta}_{k}=\min \left(0, \bar{\gamma}_{k}\right), I_{1}(a, b):=e^{b}-e^{a}, I_{2}(a, b):=$ $b e^{b}-a e^{a}-I_{1}(a, b)$ and $I_{3}(a, b):=\frac{b^{2}-a^{2}}{2}$. Assuming that $a<0<b$, then the coefficients computed with the $W A^{[a, b]}$ method are as follows,

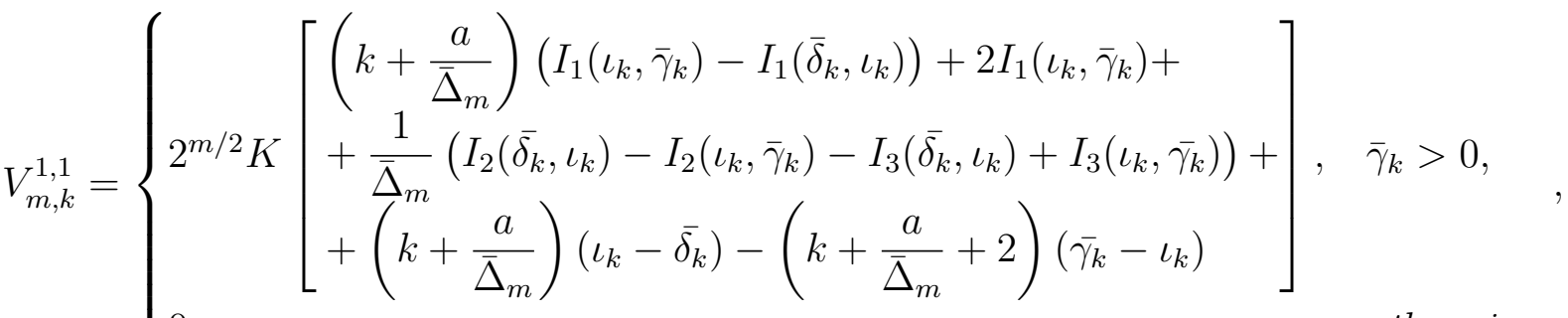
0

otherwise.

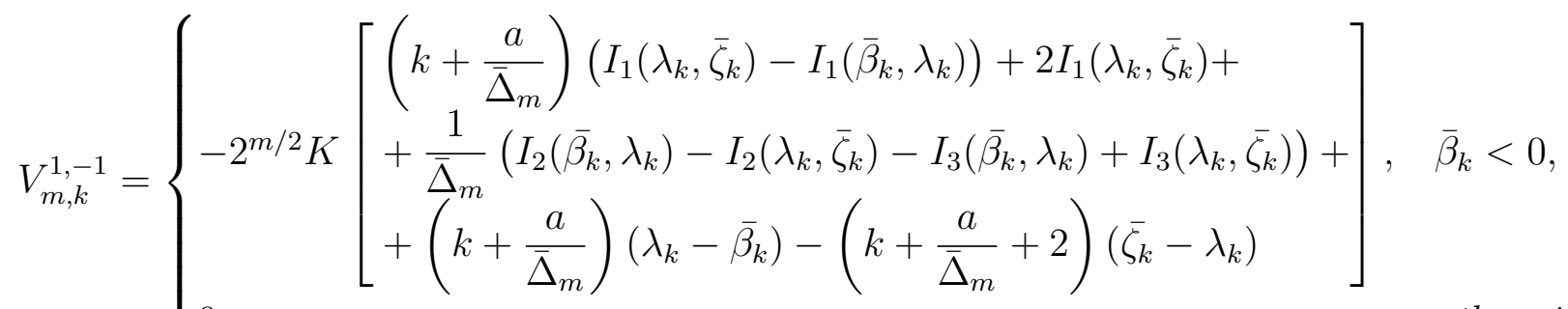
0 otherwise. for $k=0, \ldots, 2^{m+1}-2$.

If $a<b<0$, then $V_{m, k}^{1,1}=0$ and,

$$
V_{m, k}^{1,-1}=-2^{m / 2} K\left[\begin{array}{c}
\left(k+\frac{a}{\bar{\Delta}_{m}}\right)\left(I_{1}\left(\kappa_{k}, \bar{\gamma}_{k}\right)-I_{1}\left(\bar{\beta}_{k}, \kappa_{k}\right)\right)+2 I_{1}\left(\kappa_{k}, \bar{\gamma}_{k}\right)+ \\
+\frac{1}{\bar{\Delta}_{m}}\left(I_{2}\left(\bar{\beta}_{k}, \kappa_{k}\right)-I_{2}\left(\kappa_{k}, \bar{\gamma}_{k}\right)-I_{3}\left(\bar{\beta}_{k}, \kappa_{k}\right)+I_{3}\left(\kappa_{k}, \bar{\gamma}_{k}\right)\right)+ \\
+\left(k+\frac{a}{\bar{\Delta}_{m}}\right)\left(\kappa_{k}-\bar{\beta}_{k}\right)-\left(k+\frac{a}{\bar{\Delta}_{m}}+2\right)\left(\overline{\gamma_{k}}-\kappa_{k}\right)
\end{array}\right],
$$

for $k=0, \ldots, 2^{m+1}-2$. And conversely, if $0<a<b$, then $V_{m, k}^{1,-1}=0$ and,

$$
V_{m, k}^{1,1}=2^{m / 2} K\left[\begin{array}{c}
\left(k+\frac{a}{\bar{\Delta}_{m}}\right)\left(I_{1}\left(\kappa_{k}, \bar{\gamma}_{k}\right)-I_{1}\left(\bar{\beta}_{k}, \kappa_{k}\right)\right)+2 I_{1}\left(\kappa_{k}, \bar{\gamma}_{k}\right)+ \\
+\frac{1}{\bar{\Delta}_{m}}\left(I_{2}\left(\bar{\beta}_{k}, \kappa_{k}\right)-I_{2}\left(\kappa_{k}, \bar{\gamma}_{k}\right)-I_{3}\left(\bar{\beta}_{k}, \kappa_{k}\right)+I_{3}\left(\kappa_{k}, \bar{\gamma}_{k}\right)\right)+ \\
+\left(k+\frac{a}{\bar{\Delta}_{m}}\right)\left(\kappa_{k}-\bar{\beta}_{k}\right)-\left(k+\frac{a}{\bar{\Delta}_{m}}+2\right)\left(\bar{\gamma}_{k}-\kappa_{k}\right)
\end{array}\right],
$$

for $k=0, \ldots, 2^{m+1}-2$.

Proof. See Appendix B for a detailed proof.

We can also price simultaneously calls and puts, due to the compact support of B-splines scaling functions. The coefficients $V_{m, k}^{1, \alpha}$ for digital options with linear B-Splines can easily be derived following a similar procedure as in the case of Haar wavelets. Moreover, also the adaptive $\mathrm{WA}^{\mathbb{R}}$ method applies in the case of B-splines basis functions, again due to the compact support property of these functions. 


\section{ERror AnAlysis}

Let us distinguish four sources of error in our computation of price $v(x, t)$ in $(1)$ by means of the $\mathrm{WA}^{[a, b]}$ method. These are:

(A) Truncation of the integration range,

$$
\mathcal{E}_{1}(x):=v(x, t)-v^{c}(x, t)=e^{-r_{\text {int }}(T-t)} \int_{\mathbb{R} \backslash[a, b]} v(y, t) f(y \mid x) d y .
$$

(B) The approximation error at scale $m$,

$$
\mathcal{E}_{2}(x):=v^{c}(x, t)-v_{m, j}^{c}(x, t)=e^{-r_{\mathrm{int}}(T-t)} \int_{a}^{b} v(y, t)\left[f^{c}(y \mid x)-f_{m, j}^{c}(y \mid x)\right] d y .
$$

(C) The discretization error, which results when approximating the integral $I(k)$ in (14) by $I(k ; h)$ in $(15)$ using the Trapezoidal Rule. We can apply here the formula for the error of the composite Trapezoidal Rule, considering

$$
q_{m, k}^{j}(u)=\Re\left(Q_{m, j}\left(r e^{i u}\right)\right) \cos (k u), \quad \mathcal{E}_{3}:=I(k)-I(k ; h),
$$

and assuming that $q_{m, k}^{j} \in C^{2}([0, \pi])$. Then,

$$
\left|\mathcal{E}_{3}\right|=\frac{\pi^{3}}{12 M^{2}}\left|\left(q_{m, k}^{j}(\mu)\right)^{\prime \prime}\right|, \quad \mu \in(0, \pi)
$$

(D) The roundoff error. If we can calculate the sum in expression (16) with a precision of $10^{-\eta}$, then the roundoff error after multiplying by a factor $\frac{1}{M r^{k}}$ is approximately $\mathcal{E}_{4}:=\frac{1}{M r^{k}} \cdot 10^{-\eta}$. This roundoff error increases when $r$ in $(12,13,14)$ approaches 0 .

Here, we focus on the study of the error of types (A) and (B), and we refer the reader to [Ort12b] for a detailed analysis of the error of types (C) and (D).

If we define $\mathcal{E}:=v(x, t)-v_{m, j}^{c}(x, t)$, then obviously $|\mathcal{E}| \leq\left|\mathcal{E}_{1}\right|+\left|\mathcal{E}_{2}\right|$. If we assume a sufficiently wide interval $[a, b]$ for the approximation, then the overall error $\mathcal{E}$ is dominated by $\mathcal{E}_{2}$.

Looking at,

$$
\left|\mathcal{E}_{2}\right|=e^{-r_{\text {int }}(T-t)}\left|\int_{a}^{b} v(y, t)\left[f^{c}(y \mid x)-f_{m, j}^{c}(y \mid x)\right] d y\right|,
$$

then, by the Cauchy-Schwarz inequality, we have,

$$
\left|\mathcal{E}_{2}\right| \leq e^{-r_{\text {int }}(T-t)}\|v(\cdot, T)\|_{2} \cdot\left\|f^{c}(\cdot \mid x)-f_{m, j}^{c}(\cdot \mid x)\right\|_{2},
$$

where $\|\cdot\|_{2}$ indicates the norm in the $L^{2}([a, b])$ space.

Following the theory of MRA in Section 2.2, we can write,

$$
f^{c}(y \mid x)=\sum_{k=0}^{(j+1) \cdot\left(2^{m}-1\right)} c_{m, k}^{j} \phi_{m, k}^{j}\left((j+1) \cdot \frac{y-a}{b-a}\right)+\sum_{l \geq m} \sum_{k=0}^{(j+1) \cdot\left(2^{l}-1\right)} d_{l, k}^{j} \psi_{l, k}^{j}\left((j+1) \cdot \frac{y-a}{b-a}\right),
$$

where $d_{l, k}^{j}$ are the wavelet coefficients defined as,

$$
d_{l, k}^{j}:=\int_{a}^{b} f^{c}(y \mid x) \cdot \tilde{\psi}_{l, k}^{j}\left((j+1) \cdot \frac{y-a}{b-a}\right) d y,
$$

with $\tilde{\psi}^{j}$ the $j^{\text {th }}$ order dual wavelet (note that in the case of the Haar system, the $j^{\text {th }}$ order wavelet is the same as the $j^{\text {th }}$ order dual wavelet, that is, $\left.\psi^{j}=\tilde{\psi}^{j}\right)$.

The following proposition and theorem give us a bound for $\mathcal{E}_{2}$, in the case of B-splines of order zero $(j=0)$, i.e., for the Haar system. 
Proposition 3. Let us assume that $f^{c} \in C^{2}([a, b])$, and let $j=0$. If $\mathcal{M}$ is a constant such that $\left(f^{c}\right)^{\prime}(y \mid x) \leq \mathcal{M}$ for all $y \in[a, b]$, then,

$$
\left|d_{l, k}^{j}\right| \leq \frac{1}{4}(b-a)^{2} \mathcal{M} \cdot 2^{-\frac{3}{2} l}+\mathcal{O}\left(2^{-\frac{5}{2} l}\right)
$$

Proof. Let $I_{l, k}:=\left[a+k \cdot \frac{b-a}{2^{l}}, a+(k+1) \cdot \frac{b-a}{2^{l}}\right] \subset[a, b], y_{l, k}$ the midpoint of the interval $I_{l, k}$, $I_{l, k}^{w}:=\left[a+k \cdot \frac{b-a}{2^{l}}, y_{l, k}\right]$ and $I_{l, k}^{e}:=\left[y_{l, k}, a+(k+1) \cdot \frac{b-a}{2^{l}}\right]$.

We have that $f^{c}$ is twice differentiable with continuity in the interval $I_{l, k}$, and then we can expand $f^{c}$ about $y_{l, k}$ by means of Taylor's formula. It follows that for all $y \in I_{l, k}$,

$$
f^{c}(y \mid x)=f^{c}\left(y_{l, k} \mid x\right)+\left(f^{c}\right)^{\prime}\left(y_{l, k} \mid x\right)\left(y-y_{l, k}\right)+\frac{1}{2}\left(f^{c}\right)^{\prime \prime}\left(\xi_{l, k}(y) \mid x\right)\left(y-y_{l, k}\right)^{2},
$$

where $\xi_{l, k} \in I_{l, k}$.

If we substitute $f^{c}$ in (27) by its Taylor expansion and we take into account that $\psi$ has a vanishing moment of order 1 , then,

$$
d_{l, k}^{j}=\left(f^{c}\right)^{\prime}\left(y_{l, k} \mid x\right) \int_{I_{l, k}}\left(y-y_{l, k}\right) \psi_{l, k}^{j}\left(\frac{y-a}{b-a}\right) d y+\frac{1}{2} \int_{I_{l, k}}\left(f^{c}\right)^{\prime \prime}\left(\xi_{l, k}(y) \mid x\right)\left(y-y_{l, k}\right)^{2} \psi_{l, k}^{j}\left(\frac{y-a}{b-a}\right) d y .
$$

Finally, we have,

$$
\begin{aligned}
& \int_{I_{l, k}}\left(y-y_{l, k}\right) \psi_{l, k}^{j}\left(\frac{y-a}{b-a}\right) d y=2^{\frac{l}{2}} \int_{I_{l, k}^{w}}\left(y-y_{l, k}\right) d y-2^{\frac{l}{2}} \int_{I_{l, k}^{e}}\left(y-y_{l, k}\right) d y \\
& =-\frac{1}{4}(b-a)^{2} \cdot 2^{-\frac{3}{2} l}
\end{aligned}
$$

and,

$$
\begin{aligned}
& \left|\int_{I_{l, k}}\left(f^{c}\right)^{\prime \prime}\left(\xi_{l, k}(y) \mid x\right)\left(y-y_{l, k}\right)^{2} \psi_{l, k}^{j}\left(\frac{y-a}{b-a}\right) d y\right| \leq 2^{\frac{l}{2}} \max _{y \in I_{l, k}}\left|\left(f^{c}\right)^{\prime \prime}(y \mid x)\right| \int_{I_{l, k}}\left(y-y_{l, k}\right)^{2} d y, \\
& =\frac{(b-a)^{3}}{12} \max _{y \in I_{l, k}}\left|\left(f^{c}\right)^{\prime \prime}(y \mid x)\right| \cdot 2^{-\frac{5}{2} l},
\end{aligned}
$$

and this completes the proof.

Proposition 4. Under the same assumptions as Proposition 3, we have,

$$
\left|\mathcal{E}_{2}\right| \leq \mathcal{C} \sqrt{(b-a)^{5}} \cdot e^{-r_{\text {int }}(T-t)} \cdot 2^{-m} .
$$

Proof. If we consider expression (26) and we take into account that $\left\|\psi_{l, k}^{j}\left(\frac{y-a}{b-a}\right)\right\|_{2}^{2}=b-a$, then,

$$
\left\|f^{c}(\cdot \mid x)-f_{m, j}^{c}(\cdot \mid x)\right\|_{2}^{2}=(b-a) \sum_{l \geq m} \sum_{k=0}^{2^{l}-1}\left|d_{l, k}\right|^{2},
$$

since the Haar wavelets are orthogonal. By proposition 3,

$$
\left\|f^{c}(\cdot \mid x)-f_{m, j}^{c}(\cdot \mid x)\right\|_{2}^{2} \leq \frac{1}{16}(b-a)^{5} \mathcal{M}^{2} \sum_{l \geq m} 2^{-2 l}=\frac{1}{12}(b-a)^{5} \mathcal{M}^{2} 2^{-2 m} .
$$

Finally, by (25) and (28), we have,

$$
\left|\mathcal{E}_{2}\right| \leq \mathcal{C} \sqrt{(b-a)^{5}} \cdot e^{-r_{\text {int }}(T-t)} \cdot 2^{-m},
$$

and this completes the proof. 
Let us consider the $\mathrm{WA}^{\mathbb{R}}$ method. Errors of type (C) and (D) also apply in this case. Furthermore, we define,

$$
\mathcal{E}(x):=v(x, t)-v_{m, j}^{t r}(x, t)=e^{-r_{\mathrm{int}}(T-t)} \int_{\mathbb{R}} v(y, t)\left[f(y \mid x)-f_{m, j}^{t r}(y \mid x)\right] d y .
$$

Then, we have,

$$
\mathcal{E}=e^{-r_{\text {int }}(T-t)}\left[\int_{\mathbb{R} \backslash\left[\frac{k_{1}}{2^{m}}, \frac{k_{2}+1}{2^{m}}\right]} v(y, t) f(y \mid x) d y+\int_{\frac{k_{1}}{2^{m}}}^{\frac{k_{2}+1}{2^{m}}} v(y, T)\left[f(y \mid x)-f_{m, j}^{t r}(y \mid x)\right] d y\right],
$$

since $\operatorname{supp} f_{m, j}^{t r}=\left[\frac{k_{1}}{2^{m}}, \frac{k_{2}+1}{2^{m}}\right]$.

If we consider sufficiently large values $k_{1}$ and $k_{2}$, the error in (29) is mainly dominated by the second integral. Taking into account the Cauchy-Schwarz inequality, this integral can be bounded by,

$$
\begin{aligned}
& \left|\int_{\frac{k_{1}}{2^{m}}}^{\frac{k_{2}+1}{2^{m}}} v(y, T)\left[f(y \mid x)-f_{m, j}^{t r}(y \mid x)\right] d y\right| \leq\left(\int_{\frac{k_{1}}{2^{m}}}^{\frac{k_{2}+1}{2^{m}}}|v(y, T)|^{2}\right)^{2} \cdot\left(\int_{\frac{k_{1}}{2^{m}}}^{\frac{k_{2}+1}{2^{m}}}\left|f(y \mid x)-f_{m, j}^{t r}(y \mid x)\right|^{2}\right)^{2} \\
& \leq\left(\int_{\frac{k_{1}}{2^{m}}}^{\frac{k_{2}+1}{2^{m}}}|v(y, T)|^{2}\right)^{2} \cdot\left\|f(\cdot \mid x)-f_{m, j}^{t r}(\cdot \mid x)\right\|_{2},
\end{aligned}
$$

where $\|\cdot\|_{2}$ indicates the norm in the $L^{2}(\mathbb{R})$ space.

Finally, the Strang-Fix theory [Str73, Str89] states that for smooth functions,

$$
\left\|f(\cdot \mid x)-f_{m, j}^{t r}(\cdot \mid x)\right\|_{2} \leq \mathcal{D} \cdot 2^{-m(j+1)}\left\|\left(f^{c}\right)^{(j+1)}(\cdot \mid x)\right\|_{2}, \quad j \geq 0,
$$

where $\mathcal{D}$ is a constant.

Notice that in this case we obtain an estimation similar to Proposition 4 , since we can consider $\mathcal{M}=\left\|\left(f^{c}\right)^{\prime}(\cdot \mid x)\right\|_{\infty}$ in Proposition 3, where $\left\|\left(f^{c}\right)^{\prime}(\cdot \mid x)\right\|_{\infty}:=\max _{y \in[a, b]}\left|\left(f^{c}\right)^{\prime}(y \mid x)\right|$.

Remark 4. B-splines of order 1 can achieve a higher rate of convergence than B-splines of order 0. Following a similar procedure as in Proposition 3, and taking into account that B-spline wavelets have a vanishing moment of order 2 , it can be shown that the wavelet coefficients $\left|d_{l, k}^{j}\right|$ for $W A^{[a, b]}$ method are of order $2^{-3 l}$. Using this estimate, it is straightforward to proof that $\left|\mathcal{E}_{2}\right|$ is of order $2^{-2 m}$. Finally, for the $W A^{\mathbb{R}}$ method, we can also apply (30) with $j=1$.

\section{Numerical ExAmples}

In this section, we carry out several numerical experiments to evaluate the accuracy and robustness of the Wavelet Approximation method. We put our attention to plain vanilla European options with geometric Brownian motion (GBM), the Heston stochastic volatility model and the CGMY model as the associated process for the underlying asset. We will consider several maturity times, ranging from very short maturities to very long maturities, to stress the robustness of the WA method. Apart from the pricing of European options, we also perform a test to approximate a loss distribution function in a credit portfolio. This problem was treated in [Mas11] by inverting the Laplace transform of the distribution function. Here, we consider the characteristic function instead of the Laplace transform.

The recovery process of the density function is carried out by means of the Fourier inversion method presented in Section 3. We will apply two different wavelet-based approximation methods, the $\mathrm{WA}^{[a, b]}$ method and the $\mathrm{WA}^{\mathbb{R}}$ method and we will compare the results with the COS method [Fan08]. The WA ${ }^{[a, b]}$ method, as in the case of the COS method, relies in the $a$ priori computation of the approximation interval based on the cumulants for the underlying 
models. On the contrary, the $\mathrm{WA}^{\mathbb{R}}$ method computes adaptively the approximation interval to meet a predefined tolerance error in the density approximation.

To determine the interval of integration $[a, b]$ within the COS method and with the WA ${ }^{[a, b]}$ method, we consider,

$$
[a, b]:=\left[x+c_{1}-L \sqrt{c_{2}+\sqrt{c_{4}}}, x+c_{1}+L \sqrt{c_{2}+\sqrt{c_{4}}}\right], \quad \text { with } \quad x=\log \left(S_{0} / K\right) .
$$

Here, $c_{n}$ denotes the $n^{\text {th }}$ cumulant of $\log \left(S_{T} / K\right)$. The cumulants for the models employed are presented for example in [Fan08].

We set the parameters to compute the coefficients for the $\mathrm{WA}^{[a, b]}$ method at the scale of approximation $m$ as follows,

$$
r=0.9995, M=2^{m} \text {. }
$$

For a detailed description of this choice of parameters, we refer the reader to [Ort12b]. Proceeding this way, the number of coefficients used for the Haar basis is $2^{m}$ and for the B-spline basis is $2^{m+1}-1$.

We wish to show here advantages of using a wavelet method instead of the COS method. When pricing call options with the COS method, the accuracy of the solution exhibits sensitivity regarding the size of the truncated domain (31). This holds specifically for call options under fat-tailed distributions, like under certain Lévy jump processes (for instance the CGMY model), or for options with a very long time to maturity. A call payoff grows exponentially in log-asset price which may introduce cancellation errors for large domain sizes. The authors in [Fan08] use the put-call parity relation as a remedy for this, since the payoff value of a put option is bounded by the strike price. Here, we can avoid this roundoff error by means of a wavelet method without relying in the put-call parity relation. Due to the compact support of the basis functions, we can remove some of the largest payoff coefficients to achieve higher accuracy, without affecting the accuracy of the remaining part of the density. Furthermore, we can use the adaptively defined $\mathrm{WA}^{\mathbb{R}}$ method to compute the interval of the approximation, avoiding this way a strategy of fixing an interval based on cumulants.

In the following sections, we show by examples these two important issues. We use the WA $^{[a, b]}$ method to price call options under GBM and Heston model with long time to maturity and the $\mathrm{WA}^{\mathbb{R}}$ method to price a call option under the CGMY process and fat-tailed density function. It is important to underline that both wavelet methods can be applied to both situations.

5.1. Geometric Brownian Motion. A process following a GBM has a characteristic function given by,

$$
\varphi_{\mathrm{GBM}}(w)=\exp \left(-i w x-i w\left(r_{\mathrm{int}}-q-\frac{1}{2} \sigma^{2}\right)(T-t)-\frac{1}{2} \sigma^{2} w^{2}(T-t)\right) .
$$

Note that we provide a definition of the characteristic function consistent with the definition of the Fourier transform (6).

Table 1 shows the results when pricing a European call option with the WA ${ }^{[a, b]}$ method using Haar and B-splines of order 1. B-splines behave slightly better than Haar wavelets in terms of accuracy at higher scales of approximation. For shorter maturities (like the very short maturity $T=0.0001$ which is about one hour) the density function becomes extremely peaked. In this case, Haar wavelets are better than B-splines. The possible explanation is that the support for Haar basis function is half the support for B-splines scaling functions. Moreover, the shorter the maturity is, the better the accuracy for Haar wavelets. This follows from Proposition 4, since when $T \rightarrow 0$ then $b-a \rightarrow 0$. 
From now on, we will consider the Haar basis instead of the B-splines basis for the WA method, due to the fact that the WA ${ }^{[a, b]}$ method with Haar basis is more efficient and easier to implement than the same method with B-splines and the accuracy is comparable in general. It is worth mentioning that under GBM process, the COS method is extremely accurate and highly efficient, due to its exponential convergence.

\begin{tabular}{|cc|cc|cc|cc|}
\hline \hline \multicolumn{4}{|c|}{$K=120, T=0.1, L=10$} & \multicolumn{3}{c|}{$K=80, T=1, L=7.5$} \\
\hline \hline \multicolumn{2}{|c|}{ Haar } & \multicolumn{2}{c|}{ B-splines } & \multicolumn{2}{c|}{ Haar } & \multicolumn{2}{c|}{ B-splines } \\
scale & error & scale & error & scale & error & scale & error \\
\hline \hline 5 & $3.50 e-04$ & 4 & $2.60 e-04$ & 5 & $5.24 e-04$ & 4 & $1.35 e-04$ \\
6 & $9.10 e-05$ & 5 & $1.48 e-05$ & 6 & $1.02 e-04$ & 5 & $6.72 e-06$ \\
7 & $2.08 e-05$ & 6 & $3.21 e-07$ & 7 & $2.01 e-05$ & 6 & $3.61 e-07$ \\
8 & $1.41 e-06$ & 7 & $4.93 e-08$ & 8 & $4.22 e-06$ & 7 & $5.12 e-09$ \\
\hline
\end{tabular}

Table 1: Absolute errors comparing the WA ${ }^{[a, b]}$ method using Haar and B-splines basis for a European call option under GBM. Parameters: $S_{0}=100, r_{\text {int }}=0.1, q=0, \sigma=0.25$. The reference value is computed with Black-Scholes closed formulae.

We consider the $\mathrm{WA}^{\mathbb{R}}$ method to price a European call option with the Haar basis. We need an initial seed $k_{0}$ to start the algorithm. For this purpose we consider the following set of parameters,

$$
k_{0}=\left\lfloor 2^{m} \cdot a\right\rfloor, \epsilon_{\mathrm{tol}}=1 . e-15,
$$

where $a$ is defined in (31) and $L=10$. For sake of simplicity, we have taken this initial seed, although the $\mathrm{WA}^{\mathbb{R}}$ method does not rely on the a-priori choice of the interval $[a, b]$, so we can take any (random) seed to start the algorithm. Accurate results are shown in Table 2. We can observe that at scales 7 and 8 , the computed intervals for approximating the density function are almost equal. Notice that in this case the intervals are small and the density has thin tails, since it is bounded by $10^{-15}$ outside these intervals.

\begin{tabular}{|c|c|c|c|c|c|c|c|}
\hline \hline scale & $k_{1}$ & $y=\frac{k_{1}}{2^{m}}$ & $\left|f_{m, j}(y)\right|$ & $k_{2}$ & $y=\frac{k_{2}}{2^{m}}$ & $\left|f_{m, j}(y)\right|$ & error \\
\hline \hline 5 & -52 & -1.63 & $3.63 e-16$ & 21 & 0.66 & $2.49 e-16$ & $1.27 e-04$ \\
6 & -103 & -1.61 & $2.23 e-16$ & 47 & 0.73 & $2.96 e-16$ & $7.87 e-06$ \\
7 & -138 & -1.08 & $7.90 e-16$ & 64 & 0.50 & $7.59 e-16$ & $4.91 e-07$ \\
8 & -271 & -1.06 & $7.78 e-16$ & 137 & 0.54 & $6.25 e-16$ & $3.07 e-08$ \\
\hline
\end{tabular}

Table 2: Absolute errors using the $W^{\mathbb{R}}$ method for a European call option under GBM. Parameters: $S_{0}=100, K=120, r_{\text {int }}=0.1, q=0, \sigma=0.25, T=0.1$. The reference value is computed with Black-Scholes closed formulae.

We consider a European call option with a very long maturity $T=100$ (long maturities may appear for instance in economics and real options problems). Firstly, we price the option by means of the COS method using $N=32$ terms. The left plot in Figure 2 shows the payoff coefficients and the density coefficients. Due to the roundoff errors, the absolute error in the option price when comparing with the Black-Scholes closed formulae is almost $10^{+04}$. We also employ a WA ${ }^{[a, b]}$ method with the Haar basis at scale 5 (using 32 coefficients). In principle, the absolute error is, like the COS method, about $10^{+04}$. This is due to the very large payoff coefficients used in the final part of the interval (see the right plot in Figure 2). However, we can remove the last coefficients in the approximation, considering a locally truncated expansion, to achieve an accuracy of about $10^{-1}$ in the absolute error. 
We can do this because each coefficient $c_{m, k}$ in the wavelet expansion only affects the points of the density lying in an interval $\left[\frac{k}{2^{m}}, \frac{k+1}{2^{m}}\right]$. Then, for each scale level $m$, we can consider a truncated series, i.e,

$$
v(x, t) \simeq e^{-r(T-t)} \sum_{k=0}^{k(m)} c_{m, k}^{j}(x) \cdot V_{m, k}^{j, \alpha},
$$

instead of (20). We compute $k(m)$ so that $e^{-r(T-t)} \sum_{k=0}^{k(m)} c_{m, k}^{j}(x) \cdot V_{m, k}^{j, \alpha}<S_{0}$, (since $S_{0}$ is an upper bound for the value of a call) and $c_{m, k}^{j}(x)>0$ whenever $V_{m, k}^{j, \alpha}>0$ for all $k \leq k(m)$ (when the density values are close to zero, we may find some negative coefficients $c_{m, k}^{j}(x)$ that may introduce inaccurate option values).

Note that the same strategy can be followed for pricing call options with long maturities by means of the $\mathrm{WA}^{\mathbb{R}}$ method.
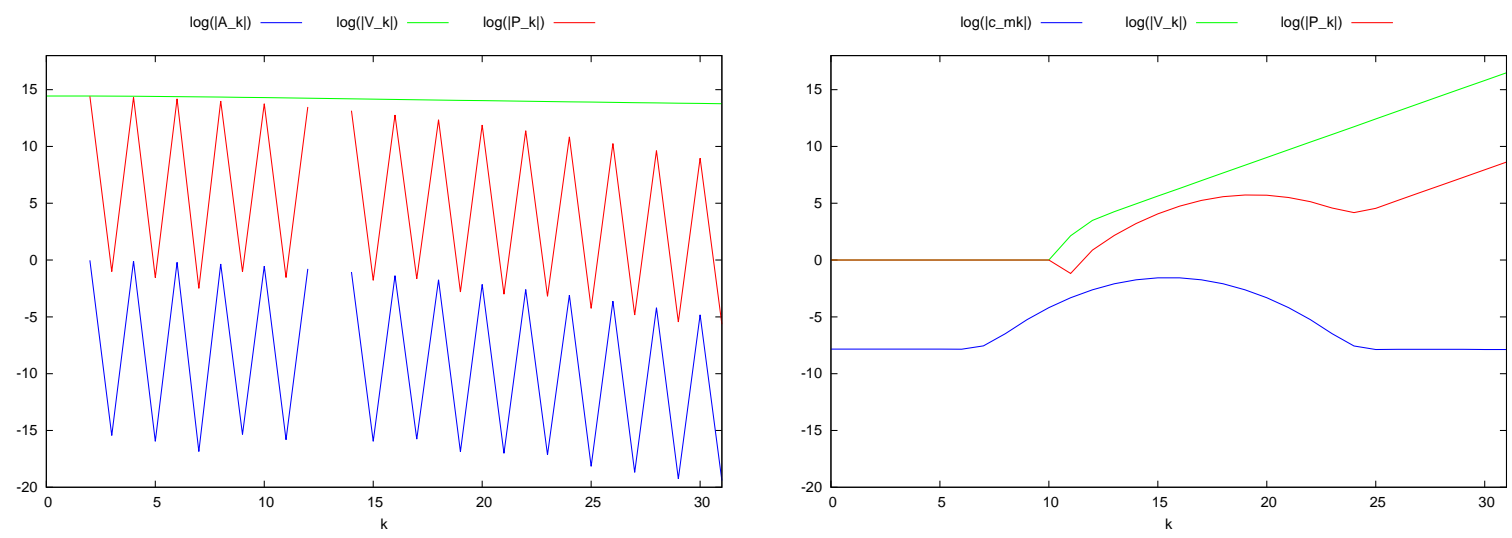

Figure 2: Payoff coefficients $\left(V_{k}\right)$ and density coefficients $\left(A_{k}\right)$ for pricing a call with the COS method (left) and coefficients $c_{5, k}^{0}, V_{5, k}^{0,1}$ for pricing a call with the WA ${ }^{[a, b]}$ method (right). Paramenters: $S_{0}=$ $100, K=100, r_{\text {int }}=0.1, q=0, \sigma=0.25, T=100, L=10$.

5.2. The Heston Model. In this section, we focus on the Heston model [Hes93] for the underlying process. Under the Heston model, the volatility, denoted by $\sqrt{u_{t}}$, is modeled by a stochastic differential equation,

$$
\left\{\begin{array}{l}
d x_{t}=\left(\mu-\frac{1}{2} u_{t}\right) d t+\sqrt{u_{t}} d W_{1 t}, \\
d u_{t}=\lambda\left(\bar{u}-u_{t}\right) d t+\eta \sqrt{u_{t}} d W_{2 t},
\end{array}\right.
$$

where $x_{t}$ denotes the log-asset price variable and $u_{t}$ the variance of the asset price process. Parameters $\lambda \geq 0, \bar{u} \geq 0$ and $\eta \geq 0$ are called the speed of mean reversion, the mean level of variance and the volatility of volatility, respectively. Furthermore, the Brownian motions $W_{1 t}$ and $W_{2 t}$ are assumed to be correlated with correlation coefficient $\rho$.

The characteristic function of the log-asset price reads,

$$
\begin{aligned}
\varphi_{\text {Heston }}(w)= & \exp (-i w x) \cdot \exp \left(-i w \mu(T-t)+\frac{u_{0}}{\eta^{2}}\left(\frac{1-e^{-D(T-t)}}{1-G e^{-D(T-t)}}\right)(\lambda+i \rho \eta w-D)\right) . \\
& \exp \left(\frac{\lambda \bar{v}}{\eta^{2}}\left((\lambda+i \rho \eta w-D)(T-t)-2 \log \left(\frac{1-G e^{-D(T-t)}}{1-G}\right)\right)\right) .
\end{aligned}
$$

We present in Table 3 the results when pricing call options with long maturities under the Heston model by means of the WA ${ }^{[a, b]}$ method with the truncated series (32) and the COS 
method. We have selected maturities $T=30$ and $T=45$ which may correspond to pension or mortgage contracts. For $T=30$ the COS method has very large errors. The WA ${ }^{[a, b]}$ method produces more accurate and robust results, in the sense that when we range from scale 6 to scale 3 , the absolute error increases up to $10^{+02}$. For the COS method, the absolute error with the same number of terms is about $10^{+06}$. For longer maturities like $T=45$, the COS method with 32 terms has an absolute error about $10^{+04}$, while the WA ${ }^{[a, b]}$ method is about half the unit. The recovered densities with the COS method are plotted in Figure 3.

\begin{tabular}{|c|c|c|c|c|c|c|c|}
\hline \multicolumn{4}{|c|}{$T=30$} & \multicolumn{4}{|c|}{$T=45$} \\
\hline \multicolumn{2}{|r|}{ Haar } & \multicolumn{2}{|r|}{$\mathrm{COS}$} & \multicolumn{2}{|c|}{ Haar } & \multicolumn{2}{|r|}{$\mathrm{COS}$} \\
\hline scale & error & $\mathrm{N}$ & error & scale & error & $\mathrm{N}$ & error \\
\hline 3 & $2.58 e+02$ & 8 & $1.72 e+06$ & 3 & $3.92 e+02$ & 8 & $3.19 e+07$ \\
\hline 4 & $2.72 e+00$ & 16 & $2.75 e+05$ & 4 & $1.09 e+01$ & 16 & $5.45 e+06$ \\
\hline 5 & $7.94 e-01$ & 32 & $2.19 e+03$ & 5 & $5.99 e-01$ & 32 & $3.10 e+04$ \\
\hline 6 & $2.46 e-03$ & 64 & $3.37 e-01$ & 6 & $1.05 e-02$ & 64 & $9.68 e-01$ \\
\hline
\end{tabular}

Table 3: Absolute errors comparing the WA ${ }^{[a, b]}$ method and the COS method for a European call option under the Heston model. Parameters: $S_{0}=100, K=100, r_{\text {int }}=0, q=0, \lambda=1.5768, \eta=0.5751, \bar{u}=$ $0.0398, u_{0}=0.0175, \rho=-0.5711, L=12$. The reference value is computed with the COS method using 50000 terms.

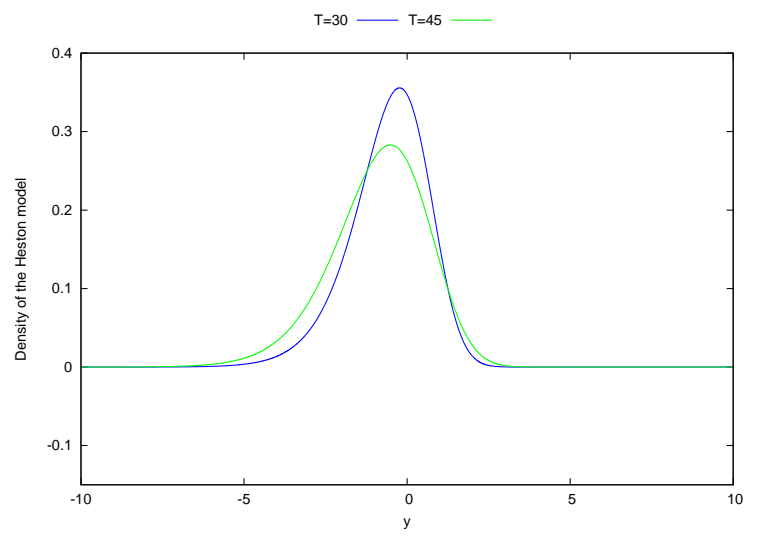

Figure 3: Recovered density funtion of the Heston model with the COS method.

5.3. CGMY Process. One problem with the GBM model is that it is not able to reproduce the volatility skew or smile present in most financial markets. Over the past few years it has been shown that several exponential Lévy models are, at least to some extent, able to reproduce the skew or the smile. One particular model is the CGMY model [Car02]. The characteristic function of the log-asset price reads,

$$
\begin{aligned}
\varphi_{\mathrm{CGMY}}(w)= & \exp (-i w x) \cdot \exp \left(-i w(r-q+s)(T-t)-\frac{1}{2} w^{2} \sigma^{2}(T-t)\right) . \\
& \exp \left(C \Gamma(-Y)\left((M+i w)^{Y}-M^{Y}+(G-i w)^{Y}-G^{Y}\right)(T-t)\right),
\end{aligned}
$$

where $\Gamma(\cdot)$ represents the Gamma function, and,

$$
s=-C \Gamma(-Y)\left((M-1)^{Y}-M^{Y}+(G+1)^{Y}-G^{Y}\right) .
$$


Here, we illustrate the sensitivity in the accuracy of the option value depending on the choice of the parameter $L$ in (31), and hence, depending on the truncation range. For this purpose, we price a call option under the CGMY process with a fat-tailed density. Figure 4 shows the price of the call option for $L$ ranging form 8 to 10 with the COS method with $N=64$ terms. We can see the fluctuation in the prices, due to cancellation errors ${ }^{1}$.

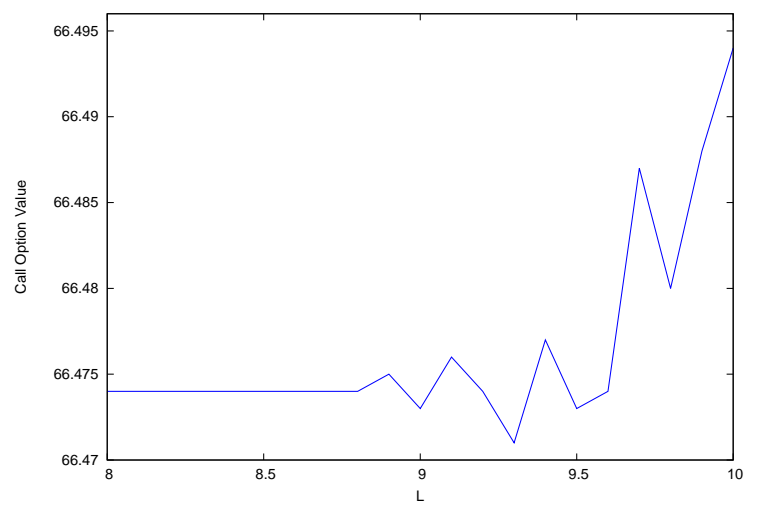

Figure 4: Call prices for the COS method under the CGMY process. Parameters: $S_{0}=100, K=$ $110, r_{\text {int }}=0.1, q=0.05, C=1, G=5, M=5, Y=1.5, T=5$.

If we consider the $\mathrm{WA}^{\mathbb{R}}$ method with $\epsilon_{\mathrm{tol}}=1 . e-15$ (for the approximation of the density), we get accurate results without relying on the choice of the interval of the approximation. The results are presented in Table 4.

\begin{tabular}{|c|c|c|c|c|c|c|c|}
\hline \hline scale & $k_{1}$ & $y=\frac{k_{1}}{2^{m}}$ & $\left|f_{m, j}(y)\right|$ & $k_{2}$ & $y=\frac{k_{2}}{2^{m}}$ & $\left|f_{m, j}(y)\right|$ & error \\
\hline \hline 2 & -109 & -27.25 & $6.29 e-16$ & 75 & 18.75 & $5.23 e-16$ & $2.83 e-05$ \\
\hline
\end{tabular}

Table 4: Absolute errors using the $W^{\mathbb{R}}$ method for a European call option under the CGMY model. Parameters: $S_{0}=100, K=110, r_{\text {int }}=0.1, q=0.05, C=1, G=5, M=5, Y=1.5, T=5$. The reference value is computed with the COS method using 50000 terms and $L=8$.

5.4. Credit Portfolio Losses. Financial companies need to evaluate and to manage risks originating from their business activities. In particular, credit risk underlying a credit portfolio is often the largest risk in a bank and its measure is used to assign capital to absorb potential losses from the credit portfolio.

Consider a portfolio consisting of $N$ obligors. The portfolio loss is defined as $L=\sum_{n=1}^{N} L_{n}$, with $L_{n}$ the individual credit loss, defined as,

$$
L_{n}=\ell_{n} \cdot \mathbf{1}_{X_{n}<c_{n}} .
$$

Here $X_{n}$ represents an individual asset return value of an obligor. So, if a companies' asset return falls below default threshold $c_{n}$, a loss $\ell_{n}$ occurs.

Capital calculations for credit portfolios are typically based on the so-called Vasicek framework [Bas05]. The Vasicek model is called a one-factor model, because the random part of the individual asset value is driven by one common economic factor, i.e.,

$$
X_{n}=\sqrt{\rho_{n}} Y+\sqrt{1-\rho_{n}} Z_{n},
$$

\footnotetext{
${ }^{1}$ By use of the put-call parity relation this may be avoided.
} 
where $Y$ is a common factor and $Z_{n}$ is the idiosyncratic factor for obligor $n$. The correlation between $\mathrm{Y}$ and $X_{n}$ is $\rho_{n}$. All random variables follow standard normal distributions, and $Y$ and $Z_{n}$ are independent.

We define $c_{n}:=\Phi^{-1}\left(P_{n}\right)$, where $\Phi^{-1}(x)$ denotes the inverse of the standard normal cumulative distribution function, and $P_{n}$ is the default probability of obligor $n$. The probability of default of obligor $n$, conditional to a realization $Y=y$, is then given by,

$$
p_{n}(y):=\mathbb{P}\left(X_{n}<c_{n} \mid Y=y\right)=\Phi\left(\frac{c_{n}-\sqrt{\rho_{n}} y}{\sqrt{1-\rho_{n}}}\right) .
$$

In the case of full granularity and for $N \rightarrow \infty$, the portfolio loss is governed by common factor $Y$, because one then assumes that the idiosyncratic factors diversify away.

When a portfolio is small or has name concentrations, e.g. when there are a few big positions so that due to the obligor's default the portfolio loss will be significant, the portfolio loss is governed by the idiosyncratic factors of these obligors. In this situation, an analytic formula is not available, and the industrial standard is to employ Monte Carlo simulations. The overall distribution function is typically a staircase-like function.

Here, however, we show that a semi-analytic formula, based on the WA ${ }^{[a, b]}$ method can be derived based on the characteristic function of the portfolio loss, which is defined as,

$$
\varphi_{\mathrm{loss}}(w)=\mathbb{E}\left[e^{-i w L}\right]
$$

The starting point for the derivation of the characteristic function is the tower property to calculate the conditional expectation, conditional on variable $Y$,

$$
\varphi_{\text {loss }}(w):=\mathbb{E}\left[\mathbb{E}\left[e^{-i w L} \mid Y\right]\right]=\mathbb{E}\left[\mathbb{E}\left[\exp \left(-i w \sum_{n=1}^{N} \ell_{n} \cdot \mathbf{1}_{X_{n}<c_{n}}\right) \mid Y\right]\right] .
$$

We recall that in a one-factor model framework, if the systematic factor $Y$ is fixed, default occurs independently since the only remaining uncertainty is the idiosyncratic risk. Then,

$$
\varphi_{\text {loss }}(w)=\mathbb{E}\left[\prod_{n=1}^{N} \mathbb{E}\left[e^{\left.-i w \ell_{n} \cdot \mathbf{1}_{X_{n}<c_{n}} \mid Y\right]}\right]=\mathbb{E}\left[\prod_{n=1}^{N} \vartheta_{n}(w ; y)\right]=\int_{\mathbb{R}} f_{Y}(y) \cdot \prod_{n=1}^{N} \vartheta_{n}(w ; y) d y\right.
$$

where,

$$
\vartheta_{n}(w ; y):=e^{-i w \ell_{n}} p_{n}(y)+1-p_{n}(y) .
$$

The conditional characteristic function for an individual obligor $\vartheta_{n}$ can be obtained analytically, and we only need to evaluate (34), the integral over $y$, numerically to find $\varphi_{\text {loss }}(w)$.

According to (33), the characteristic function $\varphi_{\text {loss }}$ is the Fourier transform of the loss density $f_{L}$ associated to the random variable $L$. Then,

$$
\varphi_{\mathrm{loss}}(w)=\int_{\mathbb{R}} e^{-i w l} f_{L}(l) d l=\int_{\mathbb{R}} e^{-i w l} F_{L}^{\prime}(l) d l,
$$

where $F_{L}^{\prime}$ is the derivative of distribution function $F_{L}$ associated to the random variable L.

Without loss of generality, we can assume that $\sum_{n=1}^{N} \ell_{n}=1$, and therefore, we can consider,

$$
F_{L}(l)= \begin{cases}\bar{F}_{L}(l), & \text { if } \quad 0 \leq l \leq 1, \\ 1, & \text { if } l>1\end{cases}
$$

for certain $\bar{F}_{L}$ defined in $[0,1]$. 
If we integrate by parts the expression (35), we have,

$$
\varphi_{\text {loss }}(w)=e^{-i w}+i w \int_{0}^{1} e^{-i w l} \bar{F}_{L}(l) d l,
$$

and then $\left(\varphi_{\text {loss }}(w)-e^{-i w}\right) /(i w)$ is the Fourier transform of $\bar{F}_{L}$.

Since $\bar{F}_{L} \in L^{2}([0,1])$, according to the theory of MRA we can approximate $\bar{F}_{L}$ in $[0,1]$ by a sum of Haar scaling functions,

$$
\bar{F}_{L}(l) \simeq \bar{F}_{L}^{m}(l), \quad \bar{F}_{L}^{m}(l)=\sum_{k=0}^{2^{m}-1} c_{m, k} \phi_{m, k}(x),
$$

with convergence in the $L^{2}$-norm. Finally, we can apply the $\mathrm{WA}^{[a, b]}$ method in a bounded interval, as described in Section 3.1, where $[a, b]=[0,1]$ in this case, to recover the coefficients of the approximation (36).

In summary, with the characteristic function of the portfolio loss determined, we can apply the WA ${ }^{[a, b]}$ method to perform the inverse Fourier transformation to recover the density or the cumulative probability function of the portfolio loss efficiently. Value-at-Risk (VaR) values can then be easily extracted from the cumulative probability function.

We consider here a very small credit portfolio with parameters $N=20, \ell_{n}=1, P_{n}=0.01$ and $\rho_{n}=0.5$ for $n=1, \ldots, N$. Figure 5 shows the tail probability of the loss random variable $L$, computed by the COS method with 1024 terms and the WA ${ }^{[a, b]}$ method at scale 10 . We use as a benchmark a Monte Carlo method with five million scenarios for the systematic factor $Y$. We can observe the high accuracy of the $\mathrm{WA}^{[a, b]}$ method at low and high loss levels. On the contrary, the COS method exhibits oscillations without additional measures and it is not capable to give reliable results at high loss levels, being often the quantiles of interest to calculate the economic capital to absorb future potential losses in the credit portfolio.
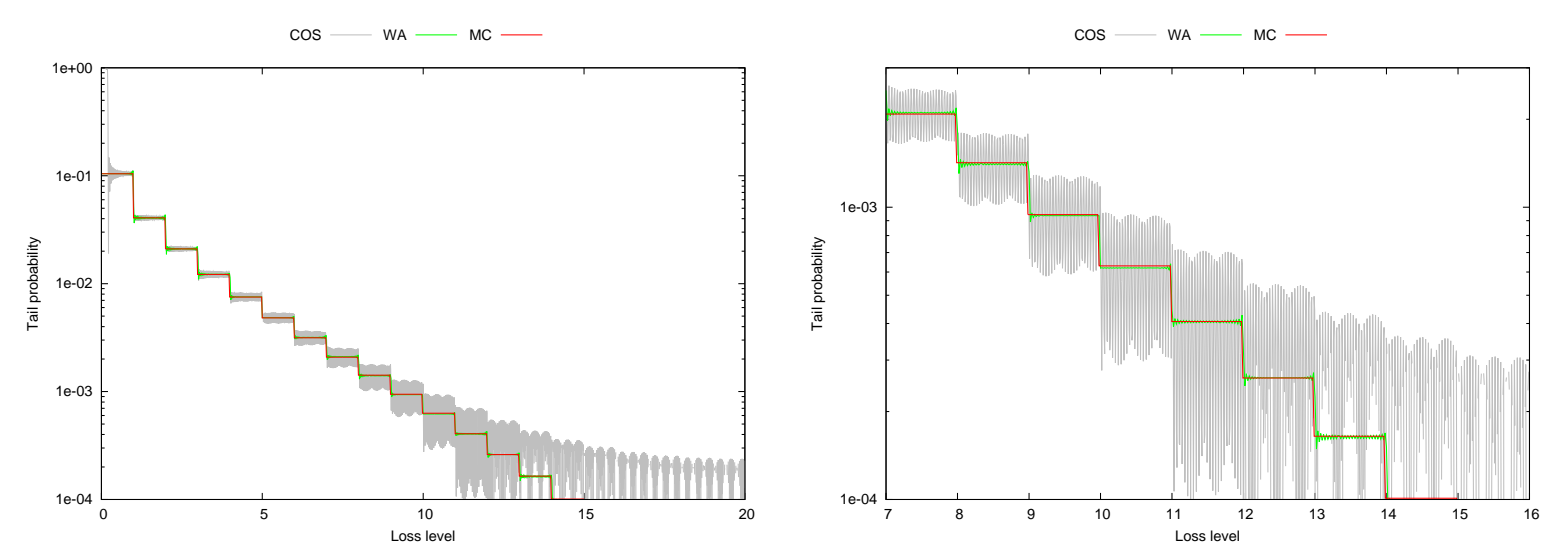

Figure 5: Tail probability of a small and diversified portfolio.

\section{Conclusions}

We have investigated option pricing problems by means of the discounted expected payoff pricing formula and the wavelet theory.

We have presented two WA methods, the $\mathrm{WA}^{[a, b]}$ and the $\mathrm{WA}^{\mathbb{R}}$ method, for recovering the density function associated to the underlying asset price process, once the characteristic function is available. Technically, we approximate the density function by a finite combination of 
$j^{\text {th }}$ order B-splines basis functions and invert its Fourier transform to recover the coefficients. With the WA ${ }^{[a, b]}$ method, we first truncate the integration range and then approximate the density function in a bounded interval. With the $\mathrm{WA}^{\mathbb{R}}$ method, we adaptively compute the integration range in order to meet a predefined tolerance error for the density approximation. The second method may be more involved, but we avoid an a-priori choice of the interval for the approximation. Regarding the selection of the basis, although in general B-splines may be somewhat more accurate than Haar wavelets, a Haar basis is more efficient and easier to implement than a B-splines basis.

We have compared the WA methods with the COS method which is based on a cosine expansion. Although the COS method is highly efficient and accurate, it may lack robustness in some cases. For long maturity contracts, the COS method may be inaccurate due to roundoff errors. With the WA method, we can truncate the series expansion conveniently to avoid roundoff errors. This is possible due to the compact support properties of the wavelets basis employed. The COS method also shows certain sensitivity on the choice of the truncation interval when pricing call options. As a remedy for this, we have proposed a solution by means of the $\mathrm{WA}^{\mathbb{R}}$ approach, avoiding the a-priori selection of the interval. It is worth mentioning that for stepped functions, like the distribution functions arising in portfolio loss problems, Haar wavelets fit much better than Fourier-based methods.

An error analysis has been presented. We underline the local convergence property of the wavelets with compact support. Due to the local nature of theses wavelets, if the approximated function is non smooth in a small interval, this does not affect the convergence in the remaining part of the approximation domain, whereas the convergence of Fourier-based approximations is affected on the whole domain.

\section{Acknowledgements}

This work was carried out during the tenure of an ERCIM "Alain Bensoussan" Fellowship Programme. The research leading to these results has received funding from the European Union Seventh Framework Programme (FP\%/200\%-2013) under grant agreement $\mathrm{n}^{\circ} 246016$. The authors wish to thank Fang Fang for pointing out the use of a characteristic function in credit portfolio loss modeling.

\section{REFERENCES}

[Bas05] Basel Committee on Bank Supervision (2005). Basel II: International convergence of capital measurement and capital standards: a revised framework.

[Bro03] M. Broadie and Y. Yamamoto (2003). Application of the fast Gauss transform to option pricing. Management Science, v. 49, 1071-1088.

[Car99] P. P. Carr and D. B. Madan (1999). Option valuation using the fast Fourier transform. Journal of Computational Finance, v. 2, 61-73.

[Car02] P. P. Carr, H. Geman, D. B. Madan and M. Yor (2002). The fine structure of asset returns: an empirical investigation. J. Business, v. 75, 305-332.

[Chu92] C. K. Chui (1992). An introduction to wavelets. Academic Press.

[Chu97] C. K. Chui (1997). Wavelets: a mathematical tool for signal analysis. SIAM Monographs on Mathematical Modeling and Computation.

[Duf00] D. Duffie, J. Pan and K. J. Singleton (2000). Transform analysis and asset pricing for affine jumpdiffusions. Econometrica, v. 68, n. 6, 1343-1376.

[Fan08] F. Fang and C.W. Oosterlee (2008). A novel pricing method for European options based on Fouriercosine series expansions. SIAM J. Sci. Comput., v. 31, 826-848.

[Fen08] L. Feng and V. Linetsky (2008). Pricing discretely monitored barrier options and defaultable bonds in Lévy process models: a fast Hilbert transform approach. Mathematical Finance, v. 18, n. 3, 337-384.

[Hes93] S. Heston (1993). A closed-form solution for options with stochastic volatility with applications to bond and currency options. Rev. Financ. Studies, v. 6, 327-343. 
[Lor07] R. Lord and C. Kahl (2007). Optimal Fourier inversion in semi-analytical option pricing. Journal of Computational Finance, v. 10, 1-30.

[Mas11] J. J. Masdemont and L. Ortiz-Gracia (2011). Haar wavelets-based approach for quantifying credit portfolio losses. Quantitative Finance, DOI: 10.1080/14697688.2011.595731.

[Mat04] A. M. Matache, T. von Petersdorff and C. Schwab (2004). Fast deterministic pricing of options on Lévy-driven assets. ESAIM: Mathematical Modelling and Numerical Analysis, v. 38, n. 1, 37-71.

[Mat05] A. M. Matache, P. A. Nitsche and C. Schwab (2005). Wavelet Galerkin pricing of American options on Lévy driven assets. Quantitative Finance, v. 5, 403-424.

[Ort12a] L. Ortiz-Gracia and J. J. Masdemont (2012). Credit risk contributions under the Vasicek one-factor model: a fast wavelet expansion approximation. To appear in Journal of Computational Finance, available at www.crm.cat.

[Ort12b] L. Ortiz-Gracia and J. J. Masdemont (2012). Peaks and jumps reconstruction with B-splines scaling functions. Working paper, available at www.crm.cat.

[Str73] G. Strang and G. Fix (1973). A Fourier analysis of the finite element variational method. Constructive Aspects of Functional Analysis, Edizioni Cremonese, Rome.

[Str89] G. Strang (1989). Wavelets and dilation equations: a brief introduction. SIAM Review, v. 31, n. 4, $614-627$.

\section{APPENDIX A}

Proof of Formula (11). We consider $P_{m, j}(z)=\sum_{l=0}^{(j+1) \cdot\left(2^{m}-1\right)} c_{m, l}^{j} z^{l}$. If we make the change of variables $z=r e^{i u}$, then,

$$
P_{m, j}\left(r e^{i u}\right)=\sum_{l=0}^{(j+1) \cdot\left(2^{m}-1\right)} c_{m, l}^{j} r^{l} e^{i l u} .
$$

Taking the real part of (37) and multiplying by $\cos (k u)$, gives us,

$$
\begin{aligned}
\Re\left(P_{m, j}\left(r e^{i u}\right)\right) \cos (k u) & =\sum_{l=0}^{(j+1) \cdot\left(2^{m}-1\right)} c_{m, l}^{j} r^{l} \cos (l u) \cos (k u) \\
& =\frac{1}{2} \sum_{l=0}^{(j+1) \cdot\left(2^{m}-1\right)} c_{m, l}^{j} r^{l}(\cos ((l+k) u)+\cos ((l-k) u)) .
\end{aligned}
$$

We integrate between 0 and $\pi$ and distinguish two cases,

- Case $k=0$ :

$$
\begin{aligned}
\int_{0}^{\pi} \Re\left(P_{m, j}\left(r e^{i u}\right)\right) & =\sum_{l=0}^{(j+1) \cdot\left(2^{m}-1\right)} c_{m, l}^{j} r^{l}\left(\int_{0}^{\pi} \cos (l u) d u\right) \\
& =\pi c_{m, 0}^{j}+\left.\sum_{l=1}^{(j+1) \cdot\left(2^{m}-1\right)} c_{m, l}^{j} r^{l} \frac{1}{l} \sin (l u)\right|_{0} ^{\pi}=\pi c_{m, 0}^{j} .
\end{aligned}
$$

- Case $k \neq 0$ :

$$
\begin{aligned}
& \int_{0}^{\pi} \Re\left(P_{m, j}\left(r e^{i u}\right)\right) \cos (k u)=\frac{1}{2} \sum_{l=0}^{(j+1) \cdot\left(2^{m}-1\right)} c_{m, l}^{j} r^{l}\left(\int_{0}^{\pi} \cos ((l+k) u) d u+\int_{0}^{\pi} \cos ((l-k) u) d u\right) \\
& =\frac{\pi}{2} c_{m, k}^{j} r^{k}+\frac{1}{2} \sum_{\substack{l=0 \\
l \neq k}}^{(j+1) \cdot\left(2^{m}-1\right)} c_{m, l}^{j} r^{l}\left(\left.\frac{1}{l+k} \sin ((l+k) u)\right|_{0} ^{\pi}+\left.\frac{1}{l-k} \sin ((l-k) u)\right|_{0} ^{\pi}\right)=\frac{\pi}{2} c_{m, k}^{j} r^{k} .
\end{aligned}
$$




\section{APPENDIX B}

Proof of Proposition 2. We consider three cases separately,

- Case $a<0<b$. We consider first the case that $\alpha=1$. By definition,

$$
V_{m, k}^{1,1}:=\int_{a}^{b}\left[K\left(e^{y}-1\right)\right]^{+} \phi_{m, k}^{1}\left(2 \cdot \frac{y-a}{b-a}\right) d y=\int_{0}^{b} K\left(e^{y}-1\right) \phi_{m, k}^{1}\left(2 \cdot \frac{y-a}{b-a}\right) d y .
$$

Taking into account that,

$$
\phi_{m, k}^{1}\left(2 \cdot \frac{y-a}{b-a}\right)=\left\{\begin{array}{ll}
2^{m / 2}\left(1-\left|2^{m+1} \cdot \frac{y-a}{b-a}-k-1\right|\right), & \frac{k}{2^{m}} \leq 2 \cdot \frac{y-a}{b-a}<\frac{k+2}{2^{m}} \\
0, & \text { otherwise. }
\end{array} .\right.
$$

we have,

$$
\begin{aligned}
& V_{m, k}^{1,1}=\int_{[0, b] \cap\left[\bar{\beta}_{k}, \bar{\gamma}_{k}\right]} 2^{m / 2} K\left(e^{y}-1\right)\left(1-\left|2^{m+1} \cdot \frac{y-a}{b-a}-k-1\right|\right) d y \\
& = \begin{cases}\int_{\bar{\delta}_{k}}^{\bar{\gamma}_{k}} 2^{m / 2} K\left(e^{y}-1\right)\left(1-\left|2^{m+1} \cdot \frac{y-a}{b-a}-k-1\right|\right) d y, & \bar{\gamma}_{k}>0 \\
0, & \text { otherwise. }\end{cases}
\end{aligned}
$$

Now, we can split the above integral in two parts yielding,

$$
\begin{aligned}
& \int_{\bar{\delta}_{k}}^{\bar{\gamma}_{k}} 2^{m / 2} K\left(e^{y}-1\right)\left(1-\left|2^{m+1} \cdot \frac{y-a}{b-a}-k-1\right|\right) d y \\
& =\int_{\bar{\delta}_{k}}^{\iota_{k}} 2^{m / 2} K\left(e^{y}-1\right)\left(2^{m+1} \cdot \frac{y-a}{b-a}-k\right) d y \\
& +\int_{\iota_{k}}^{\bar{\gamma}_{k}} 2^{m / 2} K\left(e^{y}-1\right)\left(1-2^{m+1} \cdot \frac{y-a}{b-a}+k+1\right) d y .
\end{aligned}
$$

After basic algebraic manipulation, we obtain the formulae for the coefficients $V_{m, k}^{1,1}$.

For $\alpha=-1$,

$$
V_{m, k}^{1,-1}:=\int_{a}^{b}\left[-K\left(e^{y}-1\right)\right]^{+} \phi_{m, k}^{1}\left(2 \cdot \frac{y-a}{b-a}\right) d y=\int_{a}^{0}-K\left(e^{y}-1\right) \phi_{m, k}^{1}\left(2 \cdot \frac{y-a}{b-a}\right) d y .
$$

Then,

$$
\begin{aligned}
& V_{m, k}^{1,-1}=\int_{[a, 0] \cap\left[\bar{\beta}_{k}, \bar{\gamma}_{k}\right]}-2^{m / 2} K\left(e^{y}-1\right)\left(1-\left|2^{m+1} \cdot \frac{y-a}{b-a}-k-1\right|\right) d y \\
& =\left\{\begin{array}{ll}
\int_{\bar{\beta}_{k}}^{\bar{\zeta}_{k}}-2^{m / 2} K\left(e^{y}-1\right)\left(1-\left|2^{m+1} \cdot \frac{y-a}{b-a}-k-1\right|\right) d y, & \bar{\beta}_{k}<0, \\
0, & \text { otherwise. }
\end{array} .\right.
\end{aligned}
$$

Now, we can split the above integral in two parts yielding,

$$
\begin{aligned}
& \int_{\bar{\beta}_{k}}^{\bar{\zeta}_{k}}-2^{m / 2} K\left(e^{y}-1\right)\left(1-\left|2^{m+1} \cdot \frac{y-a}{b-a}-k-1\right|\right) d y \\
& =\int_{\bar{\beta}_{k}}^{\lambda_{k}}-2^{m / 2} K\left(e^{y}-1\right)\left(2^{m+1} \cdot \frac{y-a}{b-a}-k\right) d y \\
& +\int_{\lambda_{k}}^{\bar{\zeta}_{k}}-2^{m / 2} K\left(e^{y}-1\right)\left(1-2^{m+1} \cdot \frac{y-a}{b-a}+k+1\right) d y .
\end{aligned}
$$

After basic algebraic manipulation, we obtain the formulae for the coefficients $V_{m, k}^{1,-1}$. 
- Case $a<b<0$. In this case, $\operatorname{supp} \phi_{m, k}^{1} \subset(-\infty, 0)$ and for call options $v(y, T)=$ $0, y \leq 0$. Then, $V_{m, k}^{1,1}=0, k=0, \ldots, 2^{m+1}-2$. For put options $v(y, T)>0, y \leq 0$, and therefore,

$$
\begin{aligned}
& V_{m, k}^{1,-1}=\int_{[a, b] \cap\left[\bar{\beta}_{k}, \bar{\gamma}_{k}\right]}-2^{m / 2} K\left(e^{y}-1\right)\left(1-\left|2^{m+1} \cdot \frac{y-a}{b-a}-k-1\right|\right) d y \\
& =\int_{\bar{\beta}_{k}}^{\bar{\gamma}_{k}}-2^{m / 2} K\left(e^{y}-1\right)\left(1-\left|2^{m+1} \cdot \frac{y-a}{b-a}-k-1\right|\right) d y,
\end{aligned}
$$

for $k=0, \ldots, 2^{m+1}-2$.

- Case $0<a<b$. In this case, $\operatorname{supp} \phi_{m, k}^{1} \subset(0,+\infty)$ and for call options $v(y, T)>$ $0, y>0$. Then,

$$
\begin{aligned}
& V_{m, k}^{1,1}=\int_{[a, b] \cap\left[\bar{\beta}_{k}, \bar{\gamma}_{k}\right]} 2^{m / 2} K\left(e^{y}-1\right)\left(1-\left|2^{m+1} \cdot \frac{y-a}{b-a}-k-1\right|\right) d y \\
& =\int_{\bar{\beta}_{k}}^{\bar{\gamma}_{k}} 2^{m / 2} K\left(e^{y}-1\right)\left(1-\left|2^{m+1} \cdot \frac{y-a}{b-a}-k-1\right|\right) d y,
\end{aligned}
$$

for $k=0, \ldots, 2^{m+1}-2$. For put options $v(y, T)=0, y \geq 0$, and therefore $V_{m, k}^{1,-1}=$ $0, k=0, \ldots, 2^{m+1}-2$.

Luis Ortiz-Gracia

CWI - Centrum Wiskunde \& Informatica

NL-1090 GB AMSTERDAM

The Netherlands

AND

Centre de Recerca Matemàtica

Campus de Bellaterra, Edifici C

08193 Bellaterra (BARCElona)

SPAIN

E-mail address: lortiz@crm.cat

Cornelis W. Oosterlee

CWI - Centrum Wiskunde \& Informatica

NL-1090 GB Amsterdam

The Netherlands

AND

DelfT University OF TECHNOLOGY

Delft Institute of Applied Mathematics

2628 CD DELFT

The Netherlands

E-mail address: C.W.Oosterlee@cwi.nl 